\title{
Asymmetric dark matter, inflation, and leptogenesis from $B-L$ symmetry breaking
}

\author{
Phung Van Dong* \\ Phenikaa Institute for Advanced Study, Phenikaa University, Hanoi 100000, Vietnam \\ and Faculty of Basic Science and Faculty of Materials Science and Engineering, Phenikaa University, \\ Hanoi 100000, Vietnam \\ D. T. Huong ${ }^{\dagger}$ \\ Institute of Physics, Vietnam Academy of Science and Technology, 10 Dao Tan, Ba Dinh, Hanoi, Vietnam \\ Daniel A. Camargo ${ }^{\ddagger}$ and Farinaldo S. Queiroz ${ }^{\S}$ \\ International Institute of Physics, Universidade Federal do Rio Grande do Norte, \\ Campus Universitario, Lagoa Nova, Natal-RN 59078-970, Brazil \\ José W. F. Valle \\ AHEP Group, Instituto de Física Corpuscular CSIC/Universitat de Valencia Edificio de Institutos de Paterna, \\ C/Catedratico José Beltran, 2 E-46980 Paterna (Valencia), Spain
}

(Received 9 June 2018; revised manuscript received 4 February 2019; published 27 March 2019)

\begin{abstract}
We propose a unified setup for dark matter, inflation, and baryon asymmetry generation through the neutrino mass seesaw mechanism. Our scenario emerges naturally from an extended gauge group containing $B-L$ as a noncommutative symmetry, broken by a singlet scalar that also drives inflation. Its decays reheat the universe, producing the lightest right-handed neutrino. Automatic matter parity conservation leads to the stability of an asymmetric dark matter candidate, directly linked to the matter-antimatter asymmetry in the Universe.
\end{abstract}

DOI: $10.1103 /$ PhysRevD.99.055040

\section{INTRODUCTION}

The need to account for neutrino oscillations requires new physics beyond the standard model. In addition, the cosmological challenges of particle physics, such as the need to account for dark matter, inflation, and reheating, as well as the matter-antimatter asymmetry of the Universe, all suggest the existence of new physics. Traditional proposals, based upon supersymmetry, grand unification, or extra dimensions, address only some of these issues separately. It is therefore desirable to find a comprehensive theory that can provide a common framework to address all of these puzzles.

\footnotetext{
*dong.phungvan@phenikaa-uni.edu.vn

†dthuong@iop.vast.ac.vn

†dacamargov@gmail.com

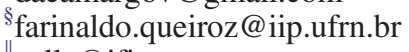

$\|_{\text {valle@ific.uv.es }}$
}

Published by the American Physical Society under the terms of the Creative Commons Attribution 4.0 International license. Further distribution of this work must maintain attribution to the author(s) and the published article's title, journal citation, and DOI. Funded by SCOAP.
The seesaw mechanism [1-9] is the most popular way to account for small neutrino masses. Here we assume that neutrino masses arise through the exchange of heavy righthanded neutrinos, hence suppressed by the right-handed mass scale. This also provides an attractive way to understand the observed baryon asymmetry of the Universe through the so-called leptogenesis mechanism [10-12]. The latter can be triggered by the $C P$-violating and out-ofthermal-equilibrium decays of the heavy right-handed neutrinos. These decays are followed by sphaleron processes in the effective standard model.

Both the seesaw mechanism and leptogenesis suggest having the $B-L$ charge as a gauge symmetry. Indeed, a gauge completion requires the presence of right-handed neutrinos as basic fermions, due to $B$ - $L$ anomaly cancellation. Moreover, $B-L$ plays a key role in converting the lepton to the baryon asymmetry during the sphaleron phase.

In addition, we also require a period of inflationary expansion of the early universe, in order to make the standard big bang picture fully consistent. This phenomenon can be parametrized by the slow-roll time evolution of a scalar field, called inflaton [13-15].

Last, but not least, the standard model lacks a stable weakly interacting massive relic that can be thermally 
produced at early times [16,17]. Moreover, the fact that dark matter searches have yielded null results, leaves the origin of dark matter as a big challenge [18-21]. Here we propose that, since dark matter and normal matter were thermally connected to begin with, they should be manifestly unified within gauge multiplets.

In this paper we show how a $B$ - $L$ gauge extension of the standard model yields a viable dark matter candidate, stabilized in a natural way by the residual matter parity which results from the extended gauge symmetry. This also fits nicely with the neutrino mass generation through the seesaw mechanism.

The latter is manifestly unified with inflation, since the inflaton $S$ arises from the field responsible for $B-L$ breaking. This takes place at a scale $\Lambda$. Right-handed neutrinos are produced via inflaton decays during reheating. As a result, in our $B-L$ gauge theory setup we have that neutrino mass generation, inflation, and reheating as well as leptogenesis are all mutually interconnected. In addition, our $B-L$ theory naturally possesses matter parity as a residual gauge symmetry. These features are in sharp contrast with the simplest Abelian $B-L$ extensions of the standard model [22-25].

In order to demonstrate all these points explicitly we start in Sec. II by describing a gauge theory that manifestly unifies the $B-L$ and electroweak charges within a 3-3-1-1 scenario in a nontrivial way. In Sec. III we describe neutrino mass generation, while in Sec. IV we examine the novel cosmological implications of our scheme concerning the issues of dark matter, inflation, and the baryon asymmetry. We conclude in Sec. V.

\section{NONCOMMUTATIVE $B-L$ DYNAMICS}

Without loss of generality, we consider only the simplest scenario, based on the 3-3-1-1 extension of the GlashowWeinberg-Salam theory [26-32]. The noncommutative $B-L$ gauge symmetry mechanism we propose is, however, more general.

The $S U(3)_{L}$ symmetry is a direct extension from the $S U(2)_{L}$ weak isospin. This extended electroweak gauge symmetry is motivated by its ability to predict the number of generations (as being equal to that of colors) as a result of $\left[S U(3)_{L}\right]^{3}$ anomaly cancellation [33].

In addition, notice that, like the electric charge $Q$, the $B$ - $L$ charge neither commutes nor closes algebraically within $S U(3)_{L}$. Hence, in order to get a consistent closed gauge structure, two new Abelian $U(1)_{X}$ and $U(1)_{N}$ gauge groups are required, where

$$
\begin{gathered}
Q=T_{3}+\beta T_{8}+X, \\
B-L=\beta^{\prime} T_{8}+N .
\end{gathered}
$$

This way we are led to the $S U(3)_{C} \otimes S U(3)_{L} \otimes U(1)_{X} \otimes$ $U(1)_{N}$ (or 3-3-1-1) group structure. Here the $T_{i}(i=$ $1,2,3, \ldots, 8)$ are the $S U(3)_{L}$ generators, while $X$, and $N$ are associated to $U(1)_{X}$, and $U(1)_{N}$, respectively. The parameters $\beta$ and $\beta^{\prime}$ are embedding coefficients, arbitrary on theoretical grounds, and independent of all anomalies. The new feature is that, in contrast to the ordinary $B-L$ symmetry, our $B-L$ is a noncommutative gauge symmetry, analogous to $Q$, nontrivially unified with the weak forces. The nontrivial commutations,

TABLE I. Field representation content of the model.

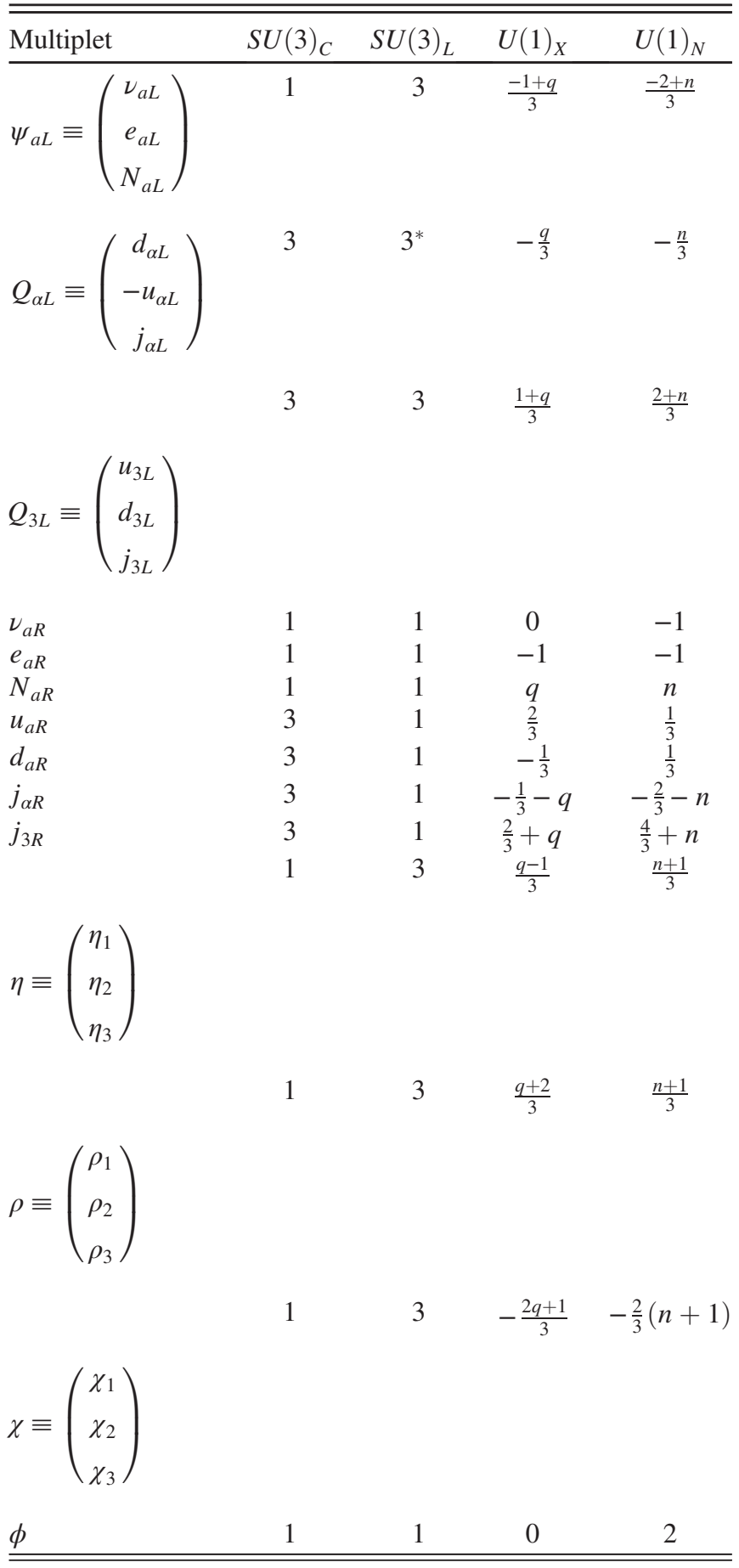


TABLE II. $Q, B-L$, and $W_{P}$ values for the model particles, where $P^{ \pm} \equiv(-1)^{ \pm(3 n+1)}$ are nontrivial for $n \neq \frac{2 m-1}{3}$. When $n=\frac{2 m}{3}, W$ particles become odd, $P^{ \pm}=-1$. The antiparticles have opposite $Q$ and $B$ - $L$, while $W_{P}$ is conjugated, $\left(P^{ \pm}\right)^{\dagger} \stackrel{3}{=} P^{\mp}$.

\begin{tabular}{lcccccccccccccccccccccccccccc}
\hline \hline Particle & $\nu_{a}$ & $e_{a}$ & $u_{a}$ & $d_{a}$ & Gluon & $\gamma$ & $W$ & $Z$ & $Z^{\prime}$ & $Z^{\prime \prime}$ & $\eta_{1}$ & $\eta_{2}$ & $\rho_{1}$ & $\rho_{2}$ & $\chi_{3}$ & $\phi$ & $N_{a}$ & $j_{\alpha}$ & $j_{3}$ & $W^{\prime}$ & $W^{\prime \prime}$ & $\eta_{3}$ & $\rho_{3}$ & $\chi_{1}$ & $\chi_{2}$ \\
\hline$Q$ & 0 & -1 & $\frac{2}{3}$ & $-\frac{1}{3}$ & 0 & 0 & 1 & 0 & 0 & 0 & 0 & -1 & 1 & 0 & 0 & 0 & $q$ & $-\frac{1}{3}-q$ & $\frac{2}{3}+q$ & $-q$ & $-1-q$ & $q$ & $1+q$ & $-q$ & $-1-q$ \\
$B-L$ & -1 & -1 & $\frac{1}{3}$ & $\frac{1}{3}$ & 0 & 0 & 0 & 0 & 0 & 0 & 0 & 0 & 0 & 0 & 0 & 2 & $n$ & $-\frac{2}{3}-n$ & $\frac{4}{3}+n$ & $-1-n$ & $-1-n$ & $1+n$ & $1+n$ & $-1-n$ & $-1-n$ \\
$W_{P}$ & 1 & 1 & 1 & 1 & 1 & 1 & 1 & 1 & 1 & 1 & 1 & 1 & 1 & 1 & 1 & 1 & $P^{+}$ & $P^{-}$ & $P^{+}$ & $P^{-}$ & $P^{-}$ & $P^{+}$ & $P^{+}$ & $P^{-}$ & $P^{-}$ \\
\hline \hline
\end{tabular}

$$
\begin{gathered}
{\left[Q, T_{1} \pm i T_{2}\right]= \pm\left(T_{1} \pm i T_{2}\right),} \\
{\left[Q, T_{4} \pm i T_{5}\right]=\mp q\left(T_{4} \pm i T_{5}\right),} \\
{\left[Q, T_{6} \pm i T_{7}\right]=\mp(1+q)\left(T_{6} \pm i T_{7}\right),} \\
{\left[B-L, T_{4} \pm i T_{5}\right]=\mp(1+n)\left(T_{4} \pm i T_{5}\right),} \\
{\left[B-L, T_{6} \pm i T_{7}\right]=\mp(1+n)\left(T_{6} \pm i T_{7}\right),}
\end{gathered}
$$

subsequently define the $Q$ and $B$ - $L$ charges for the new particles via the basic relations

$$
q \equiv-(1+\sqrt{3} \beta) / 2 \quad \text { and } n \equiv-\left(2+\sqrt{3} \beta^{\prime}\right) / 2
$$

respectively.

The simplest fermion sector, free of all gauge anomalies, is given as Table I. Notice that the scalar content is necessary for realistic symmetry breaking and mass generation. Here, $a=1,2,3$ and $\alpha=1,2$ label the particle families. Finally, $j_{a}$ and $N_{a}$ are new fermions, included in order to complete the required representations. Table II gives the $Q, B-L$ charges of the component fields.

The electrically neutral scalars can develop vacuum expectation values (vevs) given by

$$
\begin{gathered}
\langle\eta\rangle=\frac{1}{\sqrt{2}}\left(\begin{array}{l}
u \\
0 \\
0
\end{array}\right), \quad\langle\rho\rangle=\frac{1}{\sqrt{2}}\left(\begin{array}{l}
0 \\
v \\
0
\end{array}\right), \\
\langle\chi\rangle=\frac{1}{\sqrt{2}}\left(\begin{array}{l}
0 \\
0 \\
w
\end{array}\right), \quad\langle\phi\rangle=\frac{1}{\sqrt{2}} \Lambda .
\end{gathered}
$$

Here the vevs $w, \Lambda$ break the 3-3-1-1 symmetry down to the standard model times matter parity, $W_{P}=(-1)^{3(B-L)+2 s}$ (see below), providing masses to the new particles. On the other hand the vevs $u, v$ break the standard model symmetry down to $S U(3)_{C} \otimes U(1)_{Q}$, producing the ordinary particle masses.

For consistency, we impose

$$
\Lambda \gg w \gg u, v,
$$

where the first hierarchy states that the $U(1)_{N}$ breaking scale is much larger than the $S U(3)_{L} \otimes U(1)_{X}$ breaking scale, while the second hierarchy is similar to that of the simplest 3-3-1 model [33], and allows for potentially accessible new phenomena.

\section{NEUTRINO MASS GENERATION}

The above noncommutative $B-L$ dynamics provides a natural seesaw mechanism as a result of gauge symmetry breaking. We start with the implementation of the type-I seesaw mechanism (the type-II seesaw alternative in 3-3-1 models has been considered in Ref. [34].).

To analyze this we first consider the gauge symmetry breaking. This is governed by the Higgs potential, which can be separated into $V=V(\phi)+V(\eta, \rho, \chi)+V_{\text {mix }}$, where

$$
\begin{aligned}
V(\phi)= & \mu_{\phi}^{2} \phi^{\dagger} \phi+\lambda\left(\phi^{\dagger} \phi\right)^{2}, \\
V(\eta, \rho, \chi)= & \mu_{\rho}^{2} \rho^{\dagger} \rho+\mu_{\chi}^{2} \chi^{\dagger} \chi+\mu_{\eta}^{2} \eta^{\dagger} \eta+(\mu \eta \rho \chi+\text { H.c. }) \\
& +\lambda_{1}\left(\rho^{\dagger} \rho\right)^{2}+\lambda_{2}\left(\chi^{\dagger} \chi\right)^{2}+\lambda_{3}\left(\eta^{\dagger} \eta\right)^{2} \\
& +\lambda_{4}\left(\rho^{\dagger} \rho\right)\left(\chi^{\dagger} \chi\right)+\lambda_{5}\left(\rho^{\dagger} \rho\right)\left(\eta^{\dagger} \eta\right)+\lambda_{6}\left(\chi^{\dagger} \chi\right)\left(\eta^{\dagger} \eta\right) \\
& +\lambda_{7}\left(\rho^{\dagger} \chi\right)\left(\chi^{\dagger} \rho\right)+\lambda_{8}\left(\rho^{\dagger} \eta\right)\left(\eta^{\dagger} \rho\right)+\lambda_{9}\left(\chi^{\dagger} \eta\right)\left(\eta^{\dagger} \chi\right), \\
V_{\text {mix }}= & \lambda_{10}\left(\phi^{\dagger} \phi\right)\left(\rho^{\dagger} \rho\right)+\lambda_{11}\left(\phi^{\dagger} \phi\right)\left(\chi^{\dagger} \chi\right) \\
& +\lambda_{12}\left(\phi^{\dagger} \phi\right)\left(\eta^{\dagger} \eta\right),
\end{aligned}
$$

where the $\mu$-type parameters have mass dimension, while $\lambda$ 's are dimensionless.

The field $\phi$ obtains a large vev, $\Lambda^{2}=-\mu_{\phi}^{2} / \lambda$, implied by $V(\phi)$ due to $\mu_{\phi}^{2}<0, \lambda>0$. Integrating $\phi$ out, one finds that the effective potential coincides with $V(\eta, \rho, \chi)$ at the leading order. This potential provides two weak scales $u^{2}, v^{2}$ proportional to $-\mu_{\eta, \rho}^{2}>0$ and the scale $w^{2}$ proportional to $-\mu_{\chi}^{2}>0$. This is totally analogous to the situation in the 3-3-1 model. The conditions for having the above vevs amount to imposing $\left|\mu_{\phi}\right| \gg\left|\mu_{\chi}\right| \gg\left|\mu_{\eta, \rho}\right|$. Like the 3-3-1 model, a consistent Higgs boson mass spectrum can be achieved when the soft-term $\mu$ is negative at the 3-3-1 scale, i.e., $\mu<0,|\mu| \sim\left|\mu_{\chi}\right|{ }^{1}$

The Yukawa Lagrangian responsible for neutrino mass generation through the seesaw is given as

\footnotetext{
${ }^{1}$ The parameter $\mu$ can always be made real by redefining the phases of $\eta, \rho, \chi[27]$.
} 


$$
\mathcal{L} \supset h_{a b}^{\nu} \bar{\psi}_{a L} \eta \nu_{b R}+\frac{1}{2} f_{a b}^{\nu} \bar{\nu}_{a R}^{c} \nu_{b R} \phi+\text { H.c. }
$$

Note that $\phi=\frac{1}{\sqrt{2}}(\Lambda+S+i A)$ with a nonzero value for the scale $\Lambda$. Since $\phi$ has $N=B-L=2 \neq 0$, its vev breaks these charges, providing Majorana masses for $\nu_{R}$ as well as for the $U(1)_{N}$ gauge boson at the scale $\Lambda$. Since $\Lambda$ must be substantially larger than the weak scale, the $U(1)$ gauge boson is too heavy for detection. After the electroweak symmetry breaking, one generates the Dirac neutrino mass term via $\eta_{1}=\frac{1}{\sqrt{2}}\left(u+S_{1}+i A_{1}\right)$.

The total neutrino mass generation Lagrangian is

$$
\mathcal{L} \supset-\frac{1}{2}\left(\bar{\nu}_{a L} \bar{\nu}_{a R}^{c}\right)\left(\begin{array}{cc}
0 & m_{a b} \\
m_{b a} & M_{a b}
\end{array}\right)\left(\begin{array}{c}
\nu_{b L}^{c} \\
\nu_{b R}
\end{array}\right)+\text { H.c. }
$$

where $M=-f^{\nu} \Lambda / \sqrt{2}$ and $m=-h^{\nu} u / \sqrt{2}$. Since $\Lambda \gg u$, the seesaw mechanism yields the observed neutrino $\left(\sim \nu_{L}\right)$ masses in the usual manner as

$$
m_{\nu}=-m M^{-1} m^{T}=h^{\nu}\left(f^{\nu}\right)^{-1}\left(h^{\nu}\right)^{T} \frac{u^{2}}{\sqrt{2} \Lambda} .
$$

The heavy neutrinos $\left(\sim \nu_{R}\right)$ gain masses at the $B$ - $L$ breaking scale $\Lambda$. In order to obtain $m_{\nu} \sim 0.1 \mathrm{eV}$ we assume $f / h^{2} \sim 10^{14} \mathrm{GeV} / \Lambda$, since $u \sim 100 \mathrm{GeV}$. For instance, if $h, f \sim 1$ we have $\Lambda \sim 10^{14} \mathrm{GeV}$, as often assumed in the literature. However, this model prefers a larger $B-L$ breaking scale, $\Lambda>10^{14} \mathrm{GeV}$, consistent with the inflation scales. In this case $f, h$ are correspondingly adjusted, as discussed in the next section.

Since $B-L=\beta^{\prime} T_{8}+N$ annihilates the nontrivial vacua, $[B-L]\langle\eta, \rho, \chi\rangle=0$, for $u, v, w \neq 0$, it follows that the gauge group $S U(3)_{L} \otimes U(1)_{N}$ contains a residual conserved $B-L$ charge, under which a generic field transforms as

$$
\Phi \rightarrow \Phi^{\prime}=U(\omega) \Phi, \quad U(\omega)=e^{i \omega(B-L)} .
$$

However, $B-L$ is broken by $\langle\phi\rangle$ since $[B-L]\langle\phi\rangle=$ $\sqrt{2} \Lambda \neq 0$. The remnant of $B$ - $L$ preserves the vacuum, $U(\omega)\langle\phi\rangle=\langle\phi\rangle$. We obtain $e^{i \omega 2}=1$ or $\omega=m \pi$ for $m$ integer. The residual transformation is $U(m \pi)=$ $e^{i m \pi(B-L)}=(-1)^{m(B-L)}$. Multiplying $U(3 \pi)$ with spin parity $(-1)^{2 s}$ due to Lorentz symmetry, yields a matter parity $W_{P}=(-1)^{3(B-L)+2 s}$. While this is a commonly known symmetry, in our case it originates as a residual gauge symmetry,

$$
W_{P}=(-1)^{3\left(\beta^{\prime} T_{8}+N\right)+2 s},
$$

which transforms nontrivially the particles with "wrong" $B$ - $L$ charges as seen in Table II (thus the label " $W$ "). In other words, since $B-L$ is noncommutative, $W$ parity separates the gauge multiplets into two parts, including normal particles ( $W$ even) and wrong particles ( $W$ odd), respectively. (In supersymmetry, it separates supermultiplets, by contrast.) One can show that $P^{+}$and $P^{-}$particles always appear in pairs in interactions. Indeed, assume that an interaction has $x P^{+}$fields and $y P^{-}$fields. The conservation of $W$ parity implies $\left(P^{+}\right)^{x}\left(P^{-}\right)^{y}=1$, which happens only if $x=y$, for arbitrary $x, y$ integers. Thus, the lightest $W$ particle is stable and, if electrically and color neutral, can be responsible for dark matter.

The colorless $W$-particles possess electric charges $\pm q, \pm(1+q)$. Hence, we may have two dark matter options, according to whether $q=0$ and $q=-1$, or $\beta=$ $-1 / \sqrt{3}$ and $\beta=1 / \sqrt{3}$, respectively.

The model with $q=0$ yields three potential dark matter candidates, $N, W^{\prime}$, and $\eta_{3}$, whereas the model with $q=-1$ yields two possible dark matter candidates, $\rho_{3}$, and $W^{\prime \prime}$. The former has a correspondence to the original 3-3-1 model [33], while the latter does not. All of these candidates have masses proportional to the $w$ scale times the relevant coupling constants.

In this work, we consider the simplest but nontrivial case, where $q=0$ and $n=0$, hence $\beta^{\prime}=-2 / \sqrt{3}$, which has been extensively studied [26-32] under the assumption that the relics of $N, \eta_{3}$ (or $\rho_{3}$ ) were thermally produced. In such case the vectors, such as $W^{\prime}$ or $W^{\prime \prime}$, cannot be viable dark matter candidates, since they annihilate, before freezeout, into $W$ bosons via gauge self-interactions. In the present work we provide an alternative interpretation for the dark matter abundance, called asymmetric dark matter [35-37], where all possible dark matter types, including the vector one, could be viable.

\section{COSMOLOGY IN THE 3-3-1-1 MODEL}

In this section we examine how the noncommutative $B-L$ dynamics provides a natural comprehensive scenario to account for inflation, dark matter, and leptogenesis. This provides a new realization of the idea that inflation and dark matter have as common origin, the neutrino mass seesaw mechanism, proposed in Ref. [38].

Indeed, in the present context, a new superheavy Higgs scalar $S$ which breaks $U(1)_{N}$ is required, and can behave as an inflaton field, driving the early accelerated expansion of the Universe (see below). Inflaton decay only produces superheavy dark matter relics at the very large $\Lambda$ scale [29]. Fortunately, it also decays into right-handed neutrinos $\nu_{R}$, whose decays may yield $C P$-asymmetric final states consisting of normal matter $\nu_{R} \rightarrow \eta_{2} e$, as well as dark matter, $\nu_{R} \rightarrow \eta_{3} N$. The first mode yields the baryon asymmetry, while the second mode may play the main role in explaining the dark matter asymmetry, both arising from the standard leptogenesis mechanism.

The asymmetric dark matter relics may be either $\eta_{3}$ or $N$. Here, $\eta_{3}$ combines the scalar candidate, called $H^{\prime}$, and the 
Goldstone of $W^{\prime}$, called $W_{L}^{\prime}$ [27]. The present-day dark matter and normal matter relics have as a common source the right-handed $\nu_{R}$ mediating the seesaw mechanism. All candidate types, fermion $(N)$, scalar $\left(H^{\prime}\right)$, and vector $\left(W_{L}^{\prime}\right)$, can contribute to the asymmetric dark matter (a detailed evaluation is given below).

\section{A. Inflation}

In this subsection, we consider the inflationary scenario. This is linked to the singlet scalar $\phi$, which breaks the $U(1)_{N}$ symmetry. Chaotic inflation arises from a tree-level scalar potential of the type

$$
V(\phi)=\mu_{\phi}^{2} \phi^{\dagger} \phi+\lambda\left(\phi^{\dagger} \phi\right)^{2},
$$

where the scalar $\phi$ couples to additional fields such as the $U(1)_{N}$ gauge boson $(C)$, fermion fields $\left(\nu_{a R}\right)$, and scalar fields $(\eta, \rho, \chi)$. Through quantum corrections, these couplings modify the tree level inflationary potential. We denote the inflaton as $\Phi=\sqrt{2} \mathfrak{R}(\phi)$, since the imaginary part $\Im(\phi)$ is an unphysical Golstone boson that is gauged away. ${ }^{2}$ After including one-loop corrections one has [39]

$V(\Phi)=\frac{\lambda}{4}\left(\Phi^{2}-\Lambda^{2}\right)^{2}+\frac{a}{64 \pi^{2}}\left[\Phi^{4}\left(\ln \frac{\Phi^{2}}{\Lambda^{2}}-\frac{1}{2}\right)+\frac{\Lambda^{4}}{2}\right]$,

where the renormalization scale has been fixed at $\langle\Phi\rangle=$ $\Lambda=\left(-\mu_{\phi}^{2} / \lambda\right)^{1 / 2}$ satisfying the minimization condition $V^{\prime}(\Lambda)=0$, and imposing that $V(\Lambda)=0$ so as to determine the free or vacuum energy at the origin as $V_{0}=V(0)=$ $\frac{1}{4}\left(\lambda+a / 32 \pi^{2}\right) \Lambda^{4}[40-42] .^{3}$ In addition, the contributions of the scalar fields to the log term are proportional to $\lambda^{2}$ 's, hence highly suppressed if we impose $\lambda, \lambda_{10,11,12} \ll$ $g_{N}^{2},\left(f^{\nu}\right)^{2}$ as required in order to keep the flatness of the inflationary potential (see later) [28]. Therefore, the gauge and fermion contributions governing the ColemanWeinberg potential are obtained by

$$
a \simeq-\frac{1}{2} \sum_{i=1}^{3}\left(f_{i i}^{\nu}\right)^{4}+48 g_{N}^{4},
$$

where $f^{\nu}$ is taken to be flavor diagonal. Note that, with a suitable choice of the parameters, say $a / \lambda>-16 \pi^{2}$, the effective potential always has a consistent local minimum responsible for the $U(1)_{N}$ symmetry breaking. For completeness, the quantum gravity corrections to $V(\Phi)$ were

\footnotetext{
${ }^{2}$ This is in contrast to the situation considered in Ref. [38].

${ }^{3}$ Note that all the quantities appearing in the resulting potential have been renormalized at the scale $\Phi=\Lambda$, which should not be confused with the outset. Further, the couplings, e.g., $g_{N}, f^{\nu}$ disappearing inside the logarithm are due to the renormalization condition, $\left.\frac{1}{6} \frac{d^{4} V}{d \Phi^{4}}\right|_{\Phi=\Lambda}=\lambda$, or simply they are absorbed by the coupling-constant renormalization counterterm.
}

properly analyzed. They are negligible, provided that $m_{\Phi}^{2}=V^{\prime \prime}(\Phi) \ll m_{P}^{2}$ and $V(\Phi) \ll m_{P}^{4}$, where $m_{P}=$ $\left(8 \pi G_{N}\right)^{-1 / 2} \simeq 2.4 \times 10^{18} \mathrm{GeV}$ is the (reduced) Planck mass [43].

Notice that in Ref. [28] cosmic inflation was studied with an inflationary potential similar to the one in Eq. (17), but ignoring the role of the free energy $V_{0}$. Hence, the predicted results for the spectral index $n_{s}$ and tensor-to-scalar ratio $r$ and running index $\alpha$ were not fully consistent with the latest experimental results from WMAP9 and Planck [44-46]. In Ref. [29], we interpreted $V_{0}$ for multi- and single-field inflationary scenarios in another setup when the 3-3-1 breaking scale was comparable to the $U(1)_{N}$ scale. Here, we reconsider the original inflationary scenario by including the contribution of $V_{0}$, consistent with the leptogenesis scenario.

As mentioned, the spectral index $n_{s}$, the tensor-to-scalar ratio $r$, and the running index $\alpha$ are related to slow-roll parameters, $\epsilon=\frac{1}{2} m_{P}^{2}\left(V^{\prime} / V\right)^{2}, \eta=m_{P}^{2} V^{\prime \prime} / V$, and $\zeta^{2}=$ $m_{P}^{4} V^{\prime} V^{\prime \prime \prime} / V^{2}$, as follows

$n_{s} \simeq 1-6 \epsilon+2 \eta, \quad r \simeq 16 \epsilon, \quad \alpha \simeq 16 \epsilon \eta-24 \epsilon^{2}-2 \zeta^{2}$.

Further, the curvature perturbation is

$$
\Delta_{\mathcal{R}}^{2}=\frac{V}{24 \pi^{2} m_{P}^{4} \epsilon}=2.215 \times 10^{-9},
$$

at the pivot scale $k_{0}=0.05 \mathrm{Mpc}^{-1}$, and the number of $e$-folds during inflation is

$$
N=\frac{1}{\sqrt{2} m_{P}} \int_{\Phi_{e}}^{\Phi_{0}} \frac{d \Phi}{\sqrt{\epsilon}}
$$

where $\Phi_{e}$ and $\Phi_{0}$ denote the value of $\Phi$ at the end of inflation due to the violation of slow-roll condition and at the horizon exit according to $k_{0}$, respectively [15]. The value of $N$ is around 50-60 depending on the size of the inflation scale in the framework of the simplest cosmological evolution, but it may be significantly higher than that range in other cosmology scenarios [47].

One of the couplings $\lambda$ or $a$ can be appropriately fixed from the $\Delta_{\mathcal{R}}^{2}$ constraint. Hence, we are left with $r, n_{s}, \alpha$, and $N$ which are given as functions of $\Phi$ at a fixed physical scale (say $k_{0}$ ), for selected values of the remaining parameters $a / \lambda$ and $\Lambda$.

Depending on the $a / \lambda$ and $\Lambda$ values, inflation may occur as the inflaton field slowly rolls towards the potential minimum from the left $(\Phi<\Lambda)$ or the right $(\Phi>\Lambda)$. Of course, inflation terminates inducing the $U(1)_{N}$ breaking. ${ }^{4}$

\footnotetext{
${ }^{4}$ However, in the first case $\Phi<\Lambda$, if one omits $V_{0}$, the inflationary scenario seems to be excluded [28] because the predicted values of $\Delta_{\mathcal{R}}^{2}$ and $r$ are not in agreement with the observations from WMAP9 [44] and Planck $[45,46]$.
} 
TABLE III. Typical values of the parameters $\Lambda, a^{\prime}$ (thus $x_{e}, x$ ) consistent with a smallest $N$ in the allowed $n_{s}$ region; the resulting coupling $\lambda$ (thus $a$ ) from $\Delta_{\mathcal{R}}^{2}$, and the predicted observables $r, \alpha$.

\begin{tabular}{lcccccccc}
\hline \hline$m_{P} / \Lambda$ & $x_{e}$ & \multicolumn{1}{c}{$x$} & \multicolumn{1}{c}{$a^{\prime}$} & $N$ & $\lambda$ & $a$ & $r$ & $\alpha$ \\
\hline 10 & $6.6748 \times 10^{-3}$ & $6.139 \times 10^{-4}$ & $5.82 \times 10^{4}$ & 71.8 & $8.68 \times 10^{-20}$ & $1.595 \times 10^{-12}$ & $3.87 \times 10^{-12}$ & -0.000538 \\
$10^{2}$ & $5.38136 \times 10^{-4}$ & $5.26 \times 10^{-5}$ & $5.708 \times 10^{6}$ & 70.78 & $6.54 \times 10^{-22}$ & $1.178 \times 10^{-12}$ & $2.86 \times 10^{-16}$ & -0.000544 \\
$10^{3}$ & $4.64804 \times 10^{-5}$ & $4.682 \times 10^{-6}$ & $5.644 \times 10^{8}$ & 70.24 & $5.26 \times 10^{-24}$ & $0.937 \times 10^{-12}$ & $2.27 \times 10^{-20}$ & -0.000548 \\
$10^{4}$ & $4.15673 \times 10^{-6}$ & $4.264 \times 10^{-7}$ & $5.603 \times 10^{10}$ & 69.9 & $4.4 \times 10^{-26}$ & $0.779 \times 10^{-12}$ & $1.89 \times 10^{-24}$ & -0.00055 \\
\hline \hline
\end{tabular}

Thus, the inflaton field can have small or large values compared to the Planck scale during inflation corresponding to the left or right regimes, respectively. In the second case, for the large $\Phi$ field regime, the inflationary scenario merely mimics chaotic inflation, see, e.g., Refs. [38,40-42]. The latter is disfavored by current data for a convex potential like ours [46]. ${ }^{5}$ In the first case, for the small $\Phi$ field regime, the inflationary scenario follows from our noncommutative $B-L$ scenario due to the contribution of the Coleman-Weinberg potential, which is physically more attractive and unavoidable.

\section{Small-field inflation}

Here we investigate the inflationary scenario where the inflaton field starts rolling from small values, namely, $\Phi<\Lambda$, described by the full potential in Eq. (17), which sums over the leading tree-level and Coleman-Weinberg contributions,

$$
V(\Phi) \simeq V_{0}-\frac{\lambda}{2} \Lambda^{2} \Phi^{2}+\frac{a}{64 \pi^{2}} \Phi^{4}\left(\ln \frac{\Phi^{2}}{\Lambda^{2}}-\frac{1}{2}\right)
$$

Denoting $x \equiv \Phi / \Lambda<1$ and $a^{\prime} \equiv a / 32 \pi^{2} \lambda>-0.5$, the inflationary parameters or observables can be expressed as follows

$$
\begin{aligned}
\epsilon & \simeq 8\left(\frac{m_{P}}{\Lambda}\right)^{2} x^{2}\left(\frac{4 a^{\prime} x^{2} \ln x-1}{a^{\prime}+1}\right)^{2}, \\
\eta \simeq & 4\left(\frac{m_{P}}{\Lambda}\right)^{2} \frac{4 a^{\prime} x^{2}(3 \ln x+1)-1}{a^{\prime}+1}, \\
\zeta^{2} \simeq & 32\left(\frac{m_{P}}{\Lambda}\right)^{4} \\
& \times \frac{x^{2}\left(3+10 a^{\prime}+12 a^{\prime} \ln x\right)\left(4 a^{\prime} x^{2} \ln x-1\right)}{\left(a^{\prime}+1\right)^{2}}, \\
\lambda\left(a^{\prime}+1\right)^{3} \simeq & 1.67 \times 10^{-5}\left(\frac{m_{P}}{\Lambda}\right)^{6} x^{2}\left(4 a^{\prime} x^{2} \ln x-1\right)^{2},
\end{aligned}
$$

\footnotetext{
${ }^{5}$ Concerning this case, in the first version of the present work, arXiv:1805.08251v1 [hep-ph], we have shown that the new physics regime associated with the $U(1)_{N}$ symmetry lying at or above the Planck scale, is required in order to fit all the inflationary observables $[46,48,49]$. However, such a regime may be subject to quantum gravity effects (cf. Ref. [43] for details).
}

$$
N \simeq \frac{1}{4}\left(\frac{\Lambda}{m_{P}}\right)^{2}\left(a^{\prime}+1\right) \int_{x_{e}}^{x} \frac{d t}{t\left(4 a^{\prime} t^{2} \ln t-1\right)}
$$

where $x_{e} \equiv \Phi_{e} / \Lambda$, and Eq. (23) comes from the $\Delta_{\mathcal{R}}^{2}$ constraint. One sees from $V(\Phi)$ that $a^{\prime} x^{2} \ln x \sim 1$. Note also that, since $\epsilon, \eta$, and $\zeta$ have a large factor $\left(m_{P} / \Lambda\right)^{2}$, field values at the horizon exit can always be determined in the regime $\Phi_{0} / \Lambda \ll 1$. The parameters obey $\epsilon \ll|\eta| \sim|\zeta|$, thus $n_{s} \simeq 1+2 \eta, \alpha \simeq-2 \zeta^{2}$, while $r=16 \epsilon$ is strongly suppressed. Inflation ends when $\max (\epsilon,|\eta|,|\zeta|)=1$, thus $\eta=-1$. This yields

$$
4 x_{e}^{2}\left(3 \ln x_{e}+1\right) \simeq \frac{1}{a^{\prime}}-\frac{\Lambda^{2}}{4 m_{P}^{2}} \simeq-\frac{\Lambda^{2}}{4 m_{P}^{2}},
$$

where we note that $a^{\prime} \sim 1 / x^{2} \ln x \gg\left(m_{P} / \Lambda\right)^{2}$ is responsible for the small-field inflation and slow-roll condition; hence, $\Lambda / m_{P}$ governs the end of inflation.

The relation $n_{s} \simeq 1+2 \eta$ implies

$$
a^{\prime} \simeq \frac{1}{4 x^{2}(3 \ln x+1)-\left(n_{s}-1\right) \Lambda^{2} /\left(8 m_{P}^{2}\right)} .
$$

Taking $m_{P} / \Lambda=10,100,1000$, and 10000 , from Eq. (25) we obtain the corresponding values of $x_{e}$ as collected in Table III. Substituting $a^{\prime}$ and $x_{e}$ into Eq. (24), we can draw contours of $N$ as a function of $n_{s}$ and $x$ for the corresponding values of $m_{P} / \Lambda$. This is shown in various panels in Fig. 1.

That said, $n_{s}$ and $N$ would constrain $a^{\prime}, \Lambda$, while $\Delta_{\mathcal{R}}^{2}$ fixes $\lambda$ as determined by Eq. (23). One sees from the figure that the measured $n_{s}$ constrains the number of $e$-folds to be $N \geq 69.9,70.24,70.78$, and 71.8 for $\Lambda=2.4 \times 10^{14}$, $2.4 \times 10^{15}, 2.4 \times 10^{16}$, and $2.4 \times 10^{17} \mathrm{GeV}$, respectively. This does not fit well with the (upper limit) value $N \sim 60$ required for explaining the horizon problem for the case of standard cosmological evolution [47]. Indeed, $N$ depends logarithmically on the inflation scale and the reheating temperature,

$$
N \simeq 61-\ln \frac{10^{16} \mathrm{GeV}}{V_{0}^{1 / 4}}-\frac{1}{3} \ln \frac{V_{0}^{1 / 4}}{\rho_{R}^{1 / 4}},
$$

where we have used $V\left(\Phi_{e}\right) \simeq V\left(\Phi_{0}\right) \simeq V_{0}$ and the energy density at the reheating $\rho_{R} \simeq\left(\pi^{2} g_{*} / 30\right) T_{R}^{4} \lesssim V_{0}$, which are 

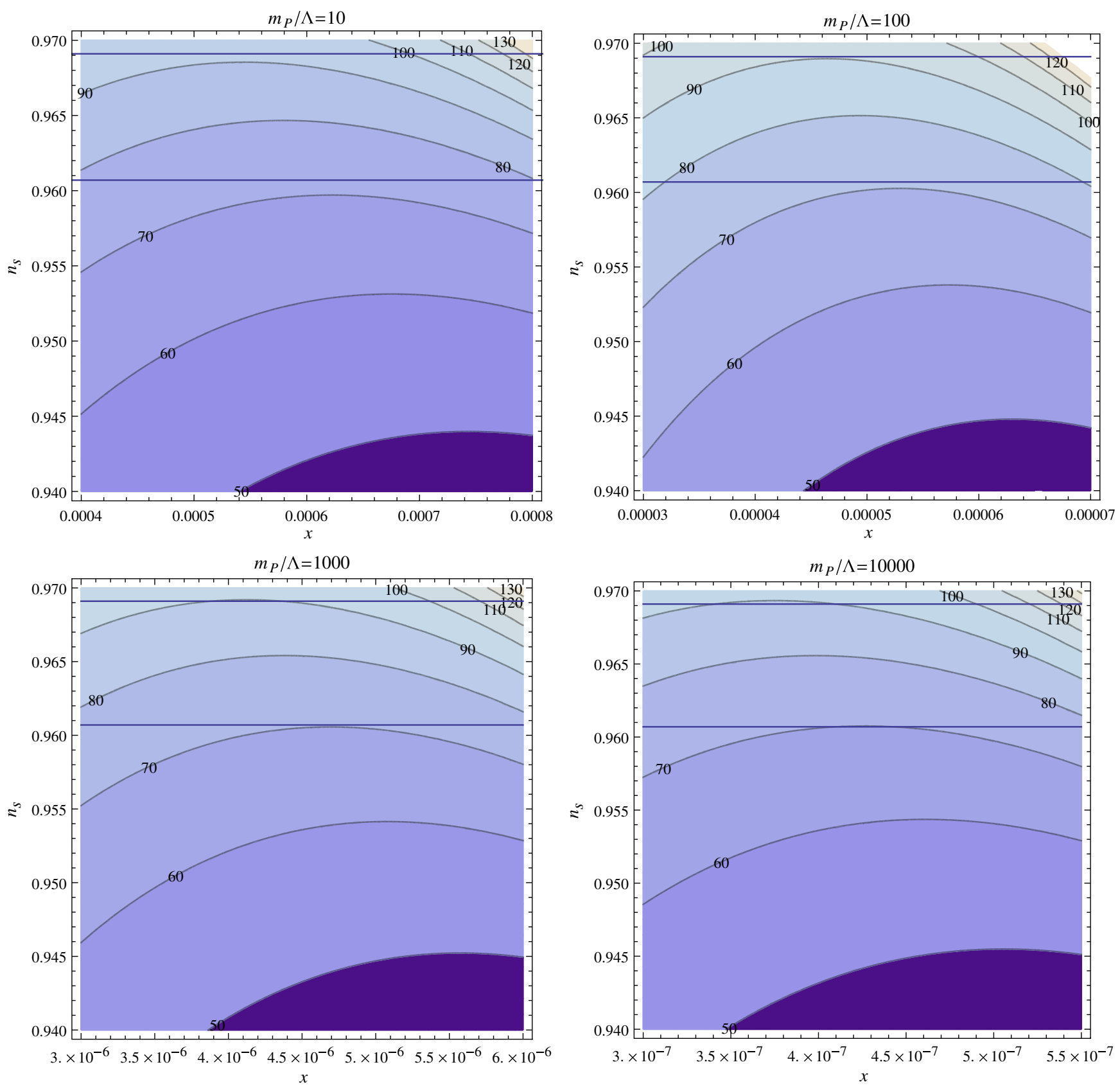

FIG. 1. Number of $e$-fold contours as a function of $n_{s}$ and $x=\Phi / \Lambda$. The various panels correspond to $m_{P} / \Lambda=10,100,1000$, and 10000 . The most recent measurement $n_{s}=0.9649 \pm 0.0042$ at $68 \%$ C.L. is also shown [50].

all ensured (details shown below). Hence, $N$ is strictly constrained, implying that this simplest scenario of smallfield inflation is ruled out.

However, this tension can be avoided by invoking new contributions. For example these can come from brane world scenarios embedded in a higher dimensional spacetime [40]. Alternatively, a fermion condensate as well as a nonminimal coupling to gravity have been suggested to avoid the problem [51,52]. Note also that the large $N$ can be reduced by introducing a second scalar field $(\chi$ or extra sextet) for the 3-3-1-1 symmetry breaking. Its unstable vacuum brings inflation quickly to an end, before breaking the slow-roll condition, as also noted in Ref. [29]. Such issues are beyond the scope of this work. Another issue that can be raised is that such approaches would change standard cosmology after inflation and right-handed neutrino production, which subsequently alters the asymmetric matter generation in the next section. Indeed, the mentioned brane world adds up to $N$ with a term $\frac{1}{2} \ln \left(1+V_{0} / 2 \Lambda_{B}\right)$, where $\Lambda_{B}$ is the brane tension. Fitting the inflationary observables prefers a low regime $V_{0}^{1 / 4} \sim 10 \mathrm{TeV}$ assuming that $\Lambda_{B}^{1 / 4}>10 \mathrm{MeV}$, but this does not exclude the large 
regime $V_{0}^{1 / 4} \sim 10^{13} \mathrm{GeV}$, given that $\Lambda_{B}^{1 / 4} \sim 10^{10} \mathrm{GeV}$. The latter may be viable for the leptogenesis. A large inflation scale and reheating temperature can emerge in the mentioned condensate scenario, since the inflaton couples both right-handed neutrinos and 3-3-1 model scalars. One type of the couplings governs the fermion condensates increasing $N$, while the other coupling type sets the reheating process, as usual.

Here we simply provide the relevant values of the parameters $x, a^{\prime}$ according to the respective smallest values of $N$ for $n_{s}=0.9607$, as listed in Table III. The table gives $\lambda$ from Eq. (23), then $a=32 \pi^{2} a^{\prime} \lambda$, and $r, \alpha$ from Eq. (19), corresponding to $m_{P} / \Lambda=10,100,1000,10000$, respectively. The effective coupling is typically $a \sim 10^{-12}$ in the whole range of $\Lambda$. The Higgs coupling is tiny, $\lambda \sim$ $10^{-19}-10^{-26}$, in order to reproduce the data after combining with the Coleman-Weinberg contributions. Otherwise, when $\lambda$ is large this case cannot explain inflation for $\Lambda<m_{P}$. The predictions of the tensor-to-scalar ratio $r \sim$ $10^{-12}-10^{-24}$ and the running index $\alpha \sim-0.0005$ agree with the previous experimental results $[44-46,48,49]$ and new update [50], say $r<0.064$ at 95\% C.L. and $\alpha=$ $-0.0045 \pm 0.0067$ at $68 \%$ C.L. Strictly speaking, the result of $r$ implies that the signals of tensor perturbations are extremely small, below the detectability limit [53]. The bounds on $\alpha$ (as well as $a, N$ ) are almost independent of the new physics scale, making our scenario testable.

Hereafter, the benchmark values of the parameters are taken as $\Lambda=2.4 \times 10^{17} \mathrm{GeV}, a \simeq 1.595 \times 10^{-12}$, and $\lambda \simeq 0.868 \times 10^{-19}$, see the first row of Table III. ${ }^{6}$ We see that the value of the inflaton field varies in the range $10^{14}-10^{15} \mathrm{GeV}$, clearly below $\Lambda$. Moreover, the interactions of $\Phi$, which induce a negative contribution to the effective potential, i.e., $a \ln (\Phi / \Lambda)<0$ or $a>0$, nicely fit the inflationary data (the sign of $a$ would be negative for the scenario of large-field inflation, previously mentioned), and the corresponding $a^{\prime}$ value satisfies the minimum condition for the full potential (17). From Eq. (18) we obtain

$$
-\sum_{i}\left(f_{i i}^{\nu} / \sqrt{2}\right)^{4}+\frac{3}{2}\left(2 g_{N}\right)^{4} \sim 10^{-12}
$$

Given that $m_{\nu_{R}}=-f^{\nu}\langle\Phi\rangle / \sqrt{2}$ and $m_{C}=2 g_{N}\langle\Phi\rangle$, and choosing reasonable values for $g_{N}$ and $f_{i i}^{\nu} \sim 10^{-3}$, one can assume the heaviest of $\nu_{i R}$ to be slightly heavier than the $U(1)_{N}$ gauge boson.

The vev of $\Phi$ arises from minimizing the scalar potential, $V^{\prime}=0$, leading to $\langle\Phi\rangle=\Lambda$. The inflaton mass and vacuum energy are estimated, respectively, as

\footnotetext{
${ }^{6}$ Note that this new physics scale is 2 orders below the Planck energy, sufficient to avoid effects of quantum gravity.
}

$$
\begin{gathered}
m_{\Phi}=\sqrt{V^{\prime \prime}(\Lambda)} \simeq \frac{1}{2 \pi} \sqrt{\frac{a}{2}} \Lambda \simeq 3.4 \times 10^{10} \mathrm{GeV}, \\
V_{0}=V(0) \simeq \frac{a}{128 \pi^{2}} \Lambda^{4} \simeq\left(4.5 \times 10^{13} \mathrm{GeV}\right)^{4},
\end{gathered}
$$

which satisfy the mentioned suppression conditions of the gravitational contribution.

Notice that the inflaton mass is much smaller than the masses of $U(1)_{N}$ gauge boson and heaviest right-handed neutrinos, since $\sqrt{a} \ll g_{N}, f_{i i}^{\nu}$ for fixing $i=2$, 3 . To make the leptogenesis mechanism viable, one assumes hierarchical Yukawa couplings

$$
f_{11}^{\nu} \sim \sqrt{a} \ll f_{22}^{\nu} \sim f_{33}^{\nu} \sim g_{N} .
$$

Correspondingly, it follows that

$$
m_{\Phi} \sim m_{\nu_{1 R}} \ll m_{\nu_{2,3 R}} \sim m_{C}
$$

The inflaton cannot decay to $U(1)_{N}$ gauge bosons nor the heavy right-handed neutrinos $\nu_{2,3 R}$. After inflation, the inflaton decays to scalars $(\rho, \eta, \chi)$ or to the lightest righthanded neutrinos $\nu_{1 R}$, which reheats the Universe, by thermalizing with the 3-3-1 model particles.

The decay rates associated to $\Phi \rightarrow \rho^{\dagger} \rho, \eta^{\dagger} \eta, \chi^{\dagger} \chi$, and $\Phi \rightarrow \nu_{1 R} \nu_{1 R}$ are given, respectively, by

$$
\Gamma_{\rho, \eta, \chi}=\frac{\lambda_{10,12,11}^{2}\langle\Phi\rangle^{2}}{16 \pi m_{\Phi}}, \quad \Gamma_{\nu_{1 R}}=\frac{\left(f_{11}^{\nu}\right)^{2} m_{\Phi}}{32 \pi} .
$$

Successful leptogenesis (see the next section) is viable for both cases, if $\Phi$ dominantly decays into a pair of scalars, while $\nu_{1 R}$ is thermally produced, or vice versa. In the first case, we must impose $\left|\lambda_{10,11,12}\right| \gg \frac{1}{4 \pi}\left|f_{11}^{\nu}\right| \sqrt{a} \sim 10^{-13}$. Since $\lambda_{10,11,12} \ll g_{N}^{2},\left(f_{22,33}^{\nu}\right)^{2} \sim 10^{-6}$, we assume $\lambda_{10,11,12} \sim$ $10^{-10}$. The reheating temperature is obtained by $\Gamma=\Gamma_{\rho}+$ $\Gamma_{\eta}+\Gamma_{\chi}$ as follows

$$
\begin{aligned}
T_{R} & =\left(\frac{90}{\pi^{2} g_{*}}\right)^{1 / 4} \sqrt{m_{P} \Gamma} \\
& \simeq 1.72 \times 10^{10}\left(\frac{\lambda_{\text {mix }}}{10^{-10}}\right)\left(\frac{10 \Lambda}{m_{P}}\right)^{1 / 2}\left(\frac{10^{-12}}{a}\right)^{1 / 4} \mathrm{GeV},
\end{aligned}
$$

where $\lambda_{\text {mix }} \equiv \sqrt{\lambda_{10}^{2}+\lambda_{11}^{2}+\lambda_{12}^{2}}$, and $g_{*}=106.75$ is the effective number of degrees of freedom. The predicted reheating temperature is $T_{R} \sim 1.72 \times 10^{10} \mathrm{GeV}$, for the typical values of the parameters. Thus, our model provides an alternative to grand unification, in which the proton is automatically stable as a result of the gauge symmetry and $W$ parity. Since supersymmetry is not invoked, we avoid the stringent bounds on the reheating temperature [54].

The lightest right-handed neutrino $\nu_{1 R}$ can be thermally generated during reheating, even though its mass is larger 
than the reheating temperature. Indeed, radiation only dominates the universe when the temperature falls below the reheating temperature. However, the inflaton decay products $\rho, \eta, \chi$ can rapidly thermalize, forming a plasma with a background temperature much higher than the reheating temperature, e.g., $10^{3} T_{R}$. As a result $\nu_{1 R}$ can be created by scattering of light states, or by thermalizing of the heavier $C, \nu_{2,3 R}[55,56]$.

For the second case, we assume that $\left|\lambda_{10,11,12}\right| \ll$ $\frac{1}{4 \pi}\left|f_{11}^{\nu}\right| \sqrt{a} \sim 10^{-13}$. The inflaton mainly decays to two $\nu_{1 R}$. The reheating temperature can be computed, yielding $T_{R} \sim 1.94 \times 10^{7} \mathrm{GeV}$, given that $f_{11}^{\nu} \sim \sqrt{a} \sim 10^{-6}$. One has a nonthermal leptogenesis mechanism as $\nu_{1 R}$ are directly produced from inflaton decays.

In the small-field inflation, the perturbative decays of the inflaton that reheat the universe may come after the preheating processes, due to the tachyonic amplification and oscillation of inflaton fluctuations, since $m_{\Phi}^{2}=$ $V^{\prime \prime}(\Phi)<0$ for $\Phi$ varying from $\Phi_{e}$ to the inflection point $\Phi_{i}<\Lambda$, where $V^{\prime \prime}\left(\Phi_{i}\right)=0$ [57]. Preheating may be proceeded by effects of parametric resonance inflaton decays, as the inflaton field couples to other scalars $\rho, \eta$, $\chi$ and is highly inhomogeneous for $\Phi>\Phi_{i}$.

\section{Large-field inflation}

In this case, the inflaton field rolls towards the potential minimum from large values, $\Phi>\Lambda$. The full potential ${ }^{7}$ is approximated as

$$
V(\Phi) \simeq \frac{1}{4}\left(\lambda+\frac{a}{8 \pi^{2}} \ln \frac{\Phi}{\Lambda}\right) \Phi^{4} \equiv \frac{1}{4} \bar{\lambda} \Phi^{4},
$$

where $\bar{\lambda}=\lambda+\left(a / 8 \pi^{2}\right) \ln (\Phi / \Lambda)$ is nearly insensitive to $\Phi / \Lambda$, which reflects a scale symmetry. Such scale invariance also prevents the quadratic term $\frac{1}{2} \mu_{\phi}^{2} \Phi^{2}$ and the free energy $V_{0}$ from turning on. Recall that even without scale symmetry this case cannot fit the inflation data for $\Lambda$ below $m_{P}$. However, it does so if $\Lambda$ is at or above $m_{P}$ with inclusion of $V_{0}$, which is theoretically disfavored. Indeed, since in the Planck regime the effect of quantum gravity may become important, the standard QFT computations dealing with pointlike particles are ill defined due to the nonrenormalizability of gravity. Hence, we safely impose $\Lambda<m_{P}$, as in the previous case.

For the large field inflation, we can make use of the effective field theory (validated later). Ignoring gravity, we have the Coleman-Weinberg effective potential where the renormalization scale is fixed as $\Lambda$ below the Planck scale which, as stated, approximately conserves scale symmetry. Including gravity, the Lagrangian has a nonminimal coupling of the inflaton to gravity, $\mathcal{L} \supset \frac{1}{2}\left(m_{P}^{2}+\xi \Phi^{2}\right) R$, where

\footnotetext{
${ }^{7}$ Note that its parameters are not restricted by the previous constraints.
}

$R$ is the scalar curvature, and $\xi$ satisfies $1 \ll \xi \ll\left(m_{P} / \Lambda\right)^{2}$ in order to maintain the chaotic inflation and keep consistent Higgs physics from induced gravity. Generalizing the results in Refs. [58,59], we achieve (i) conformally transforming to the Einstein frame $\hat{g}_{\mu \nu}=\Omega^{2} g_{\mu \nu}$ with $\Omega^{2}=1+\xi \Phi^{2} / m_{P}^{2}$. The effective potential induced by gravity takes the form,

$$
U(\hat{\Phi})=\frac{V}{\Omega^{4}} \simeq \frac{\bar{\lambda} m_{P}^{4}}{4 \xi^{2}}\left(1-e^{-\sqrt{\frac{2}{3} \hat{\omega}}}\right)^{2}
$$

at the leading order, where $\hat{\Phi}$ is the canonically normalized inflaton field, related to the original field by $\hat{\Phi} \simeq$ $\sqrt{3 / 2} m_{P} \ln \Omega^{2}$. (ii) This potential is flat for large field values, $\hat{\Phi} \gg m_{P}$ (or $\Phi \gg m_{P} / \sqrt{\xi}$ ), which successfully fits inflationary observables, i.e., $n_{s}=0.967, r=0.003$, and $\alpha=-5 \times 10^{-4}$, given that $N=62$ and $\xi / \sqrt{\bar{\lambda}} \simeq 4.9 \times 10^{4}$, where the last one is fixed by the curvature perturbation.

The $\xi$ condition translates to $4.16 \times 10^{-10} \ll \bar{\lambda} \ll$ $0.0416\left(m_{P} / 100 \Lambda\right)^{4}=4.16 \times 10^{-6}, 4.16 \times 10^{-2}$, according to $m_{P} / \Lambda=10,100$, respectively. Taking $\Lambda=2.4 \times$ $10^{17} \mathrm{GeV}$ as in the previous section, ${ }^{8}$ one gets roundly $\bar{\lambda} \simeq \lambda \sim 10^{-8}$, assuming $a \lesssim \lambda$ to keep both the flatness of the inflationary potential and the insensitivity of $\bar{\lambda}$ when the nonminimal coupling contributes. The last condition leads to $g_{N}, f_{i i}^{\nu} \lesssim 10^{-2}$. Since $\rho, \eta, \chi$ potentially contribute to $a$ [28], we further require $\sqrt{\lambda}_{10,11,12} \lesssim g_{N}, f_{i i}^{\nu}$. Let us stress that the Coleman-Weinberg contributions to the nonminimal scalar coupling are suppressed by large $m_{P}$ and $\xi$ (see, for instance, Ref. [59]). That said, the nonminimal coupling improves the result of the chaotic inflation, which does not require too small $\lambda, a$ couplings as in the previous section. When $\xi \simeq 1$, the corrections of order $m_{P}^{2} / \xi \Phi^{2}$ are not negligible, which will not be considered in this work. When $\xi \ll 1$, the gravitational effect on inflation is negligible. Last but not least, the effect of quantum gravity that contributes to the inflationary potential is proportional to $U(\hat{\Phi}) / m_{P}^{4} \sim \bar{\lambda} / \xi^{2} \sim 10^{-10}$, which is strongly suppressed, justifying the effective theory of large field inflation $[43,58]$.

After inflation, the inflaton oscillates near the single minimum and violates the scale symmetry. This is governed by the vacuum energy $V_{0}$ and the soft $\mu_{\phi}$ term plus a quadratic term present when expanding $U(\hat{\Phi})$, which yields

$$
U(\hat{\Phi})=\frac{1}{2} m_{\hat{\Phi}}^{2}(\hat{\Phi}-\hat{\Lambda})^{2}+\mathcal{O}\left(\hat{\Phi}^{3}\right)
$$

where we have used $V_{0} \simeq(\bar{\lambda} / 4) \Lambda^{4}, \quad \mu_{\phi}^{2} \simeq-\bar{\lambda} \Lambda^{2}$ and $\hat{\Lambda} \equiv \xi \sqrt{3 / 2} \Lambda^{2} / m_{P}$. The inflaton mass is fixed at $m_{\hat{\Phi}}=\sqrt{\bar{\lambda} / 3} m_{P} / \xi \simeq 2.8 \times 10^{13} \mathrm{GeV}$. The higher-order

\footnotetext{
${ }^{8}$ In this case, the $\xi$ regime is narrow but viable, e.g., $\xi \sim 10$.
} 
corrections proportional to $m_{\hat{\Phi}}^{2} m_{P}^{2} \mathcal{O}\left[\left(\hat{\Phi} / m_{P}\right)^{3}\right]$ will be quickly negligible after the end of inflation. It is noteworthy that the inflaton does not couple to the standard model gauge bosons and fermions, which yields a novel scenario for (p)reheating, in contrast to the standard scenario [59]. Let us see.

Appropriate to the leptogenesis scenario, we assume $f_{11}^{\nu} \sim \sqrt{\lambda}_{10,11,12} \ll f_{22,33}^{\nu} \sim g_{N} \lesssim 10^{-2}$. In the Einstein frame, we have as a result that $m_{\nu_{1 R}} \sim m_{\rho, \eta, \chi} \ll$ $m_{\nu_{2,3 R}} \sim m_{C} \lesssim 10^{-2}\left(\sqrt{2 / 3} m_{P} \hat{\Phi} / \xi\right)^{1 / 2}$, where $m_{C}=2 g_{N} \times$ $\left(\sqrt{2 / 3} m_{P} \hat{\Phi} / \xi\right)^{1 / 2}, \quad m_{\nu_{i R}}=-f_{i i}^{\nu}\left(m_{P} \hat{\Phi} / \sqrt{6} \xi\right)^{1 / 2}, \quad$ and $m_{\rho, \chi, \eta}=\sqrt{\lambda}_{10,11,12}\left(m_{P} \hat{\Phi} / \sqrt{6} \xi\right)^{1 / 2}$, respectively. Here, $\hat{\Phi}=\hat{\Phi}(t) \simeq\left(m_{P} / m_{\Phi} t\right) \sin \left(m_{\Phi} t\right)+\hat{\Lambda}$ is the approximate solution of the Klein-Gordon equation for the inflaton field, rolling within the range $\hat{\Phi}_{e}>\hat{\Phi}>\hat{\Lambda}$. Note that the inflation ends at $\epsilon\left(\hat{\Phi}_{e}\right)=1$ implying $\hat{\Phi}_{e} \simeq 0.4 m_{P}$, and the vev of $\hat{\Phi}$ is $\hat{\Lambda} \simeq 0.06 m_{P}$. Thus, the inflaton needs about 10 semi-oscillations after inflation to reach the potential minimum. The perturbative decays are allowed for $m_{\Phi}>2 m_{\nu_{i R}}, 2 m_{C}, 2 m_{\rho, \eta, \chi}$, leading to $\hat{\Phi}<(1 / 4) \sqrt{\bar{\lambda} / 2} m_{\Phi} / h^{2} \sim\left(4.2 \times 10^{-3} / h\right)^{2} m_{\Phi}$ for a common coupling $h=-f_{i i}^{\nu} / \sqrt{2}, 2 g_{N}$, or $\sqrt{\lambda_{10,11,12} / 2}$, respectively. Comparing the upper limit with $\hat{\Phi}_{e}$, it follows that the inflaton field immediately decays (just after the inflation), since $h<2.26 \times 10^{-5}$. Let the couplings arrange as $f_{11}^{\nu}, \sqrt{\lambda}_{10,11,12}<2.26 \times 10^{-5} \ll f_{22,33}^{\nu}, g_{N} \lesssim 10^{-2}$. The viable channels are $\hat{\Phi} \rightarrow \nu_{1 R} \nu_{1 R}, \eta^{\dagger} \eta, \rho^{\dagger} \rho, \chi^{\dagger} \chi$. The inflaton cannot decay perturbatively to $\nu_{2,3 R}$ and $C$ after the ten semi-oscillations and after approaching $\hat{\Phi} \rightarrow \hat{\Lambda}$, since these are kinematically forbidden. As in the previous section, the reheating temperature is evaluated to be $T_{R} \sim 6 \times 10^{8} \mathrm{GeV}$ $\left(5.5 \times 10^{9} \mathrm{GeV}\right)$, corresponding to the products $\rho, \eta, \chi$ $\left(\nu_{1 R}\right)$ with the choice $\sqrt{\lambda}_{10,11,12} \sim 10^{-5}\left(f_{11}^{\nu} \sim 10^{-5}\right)$, respectively.

Moreover, the Hubble rate can be estimated as $H=\frac{1}{\sqrt{3} m_{P}} \sqrt{\rho}_{\hat{\Phi}} \simeq 0.13 m_{\hat{\Phi}} j^{-1}$, where $j \equiv m_{\hat{\Phi}} t / \pi$ is the number of inflaton semi-oscillations after inflation. Comparing to the typical decay rate, $H \sim \Gamma \simeq$ $\left(h^{2} / 16 \pi\right) m_{\hat{\Phi}}$, the inflaton needs $2 j \sim 10^{11}$ oscillations in order for their decay products to thermalize, which is common in inflation models. In this stage of preheating, although the perturbative decay $\hat{\Phi} \rightarrow C C$ is prevented, the quantum fields $C$ may be generated by a broad parametric resonance due to the gauge interaction $2 g_{N}^{2} C^{2} \hat{\Phi}^{2}$, characterized by the parameter $q_{b} \equiv g_{N}^{2}|\hat{\Phi}|^{2} / m_{\hat{\Phi}}^{2} \sim 10^{4}-10^{2}$ when the inflaton oscillation amplitude $|\hat{\Phi}|=m_{P} / \pi j$ varies from the end of inflation until 10 semi-oscillations; see Refs. [60-62]. And, when the number of oscillations is large enough, the narrow parametric resonance $q_{n} \equiv$ $16 g_{N}^{2}|\hat{\Phi}| \hat{\Lambda} / m_{\hat{\Phi}}^{2} \lesssim 1$ with $|\hat{\Phi}| \ll \hat{\Lambda}$ may happen for
$\hat{\Phi} \rightarrow C C$. All those lead to the exponential growth of generated $C$ 's, but the products are correspondingly diluted due to the Hubble expansion, the decay of $C$ 's to the righthanded neutrinos and the 3-3-1 model particles (including ordinary quarks and leptons), as well as the back reaction of $C$ 's into inflatons. Such a preheating process can alter the perturbative decays and significantly raise the reheating temperature. Note that the nonperturbative parametric resonance effects cannot happen for the fermion products, $\hat{\Phi} \rightarrow \nu_{i R} \nu_{i R}$, due to the Pauli exclusion principle. Moreover, the processes $\hat{\Phi} \rightarrow \rho^{\dagger} \rho, \eta^{\dagger} \eta, \chi^{\dagger} \chi$ may also arise nonperturbatively by narrow parametric resonances. In short, the preheating phases of the present and previous sections are quite rich and worth exploring, a task to be taken up elsewhere.

In what follows we will take both small- and large-field parameter regimes into account, but the results obtained are general. Note that, after the large-field inflation, $\Omega^{2} \rightarrow 1$, the scale parameters and fields approximate the usual ones in the Jordan frame, used hereafter. To close this section, it is useful to summarize the benchmark values adopted for the various parameters, including masses and couplings, as Table IV. For convenience, this table also collects those in other sections.

\section{B. Leptogenesis: Normal vs dark matters}

One of the most attractive features of the current model lies in the lepton sector. The right-handed neutrinos are singlets under $S U(3)_{L} \otimes U(1)_{X}$, but transform nontrivially under $U(1)_{N}$. Since they carry one unit of $B-L$, they acquire Majorana mass (two units of lepton number) due to $U(1)_{N}$ breaking. This constitutes a source for lepton and dark matter asymmetries in the model.

The relevant Lagrangian is given by

$$
\begin{aligned}
\mathcal{L} \supset & -\bar{e}_{a L}\left(m_{e}\right)_{a b} e_{b R}-\bar{N}_{a L}\left(m_{N}\right)_{a b} N_{b R}-\frac{1}{2} \bar{\nu}_{a R}^{c} M_{a b} \nu_{b R} \\
& +h_{a b}^{\nu}\left(\bar{e}_{a L} \eta_{2}^{-}+\bar{N}_{a L} \eta_{3}^{0}\right) \nu_{b R}+\text { H.c. }
\end{aligned}
$$

where the mass matrices, $m_{e}=-h^{e} v / \sqrt{2}$ and $m_{N}=$ $-h^{N} w / \sqrt{2}$, arise from the Yukawa interactions, $h_{a b}^{e} \bar{\psi}_{a L} \rho e_{b R}+h_{a b}^{N} \bar{\psi}_{a L} \chi N_{b R}$, respectively [31], while the other terms come from the above seesaw mechanism. The gauge states $\left({ }_{a}\right)$ are related to the mass eigenstates $\left({ }_{i}\right)$ by mixing matrices, $e_{a L, R}=\left(V_{e L, R}\right)_{a i} e_{i L, R}$, $N_{a L, R}=\left(V_{N L, R}\right)_{a i} N_{i L, R}$, and $\nu_{a R}=\left(V_{\nu R}\right)_{a i} \nu_{i R}$, which so that $\quad V_{e L}^{\dagger} m_{e} V_{e R}=\operatorname{diag}\left(m_{e}, m_{\mu}, m_{\tau}\right), \quad V_{N L}^{\dagger} m_{N} V_{\mathrm{NR}}=$ $\operatorname{diag}\left(m_{N_{1}}, m_{N_{2}}, m_{N_{3}}\right)$, and $V_{\nu R}^{T} M V_{\nu R}=\operatorname{diag}\left(M_{1}, M_{2}, M_{3}\right)$, respectively, leading to

$$
\mathcal{L} \supset \cdots-\frac{1}{2} M_{i} \bar{\nu}_{i R}^{c} \nu_{i R}+x_{i j} \bar{e}_{i L} \eta_{2}^{-} \nu_{j R}+y_{i j} \bar{N}_{i L} \eta_{3}^{0} \nu_{j R}+\text { H.c. }
$$


TABLE IV. Benchmark parameter values used or determined throughout the text, where we note that the model can work for a much smaller $\Lambda$ and a larger $w$ (see the text in detail).

\begin{tabular}{lcccc}
\hline \hline$Q, B$ - $L$ embedding & $\beta=-\frac{1}{\sqrt{3}}\left(\frac{1}{\sqrt{3}}\right)$ & $q=0(-1)$ & $\beta^{\prime}=-\frac{2}{\sqrt{3}}$ & $n=0$ \\
Breaking scales (vev) & $u \sim 100 \mathrm{GeV}$ & $v \sim 100 \mathrm{GeV}$ & $w \sim 5 \mathrm{TeV}$ & $\Lambda \simeq 2.4 \times 10^{17} \mathrm{GeV}$ \\
Small-field inflation & $g_{N} \sim f_{22,33}^{\nu} \sim 10^{-3}$ & $f_{11}^{\nu} \sim 10^{-6}$ & $\lambda \simeq 0.868 \times 10^{-19}$ & $\lambda_{10,11,12} \sim 10^{-10}\left(\ll 10^{-13}\right)$ \\
& $m_{\Phi} \simeq 3.4 \times 10^{10} \mathrm{GeV}$ & $m_{\nu_{1 R}} \sim 10^{10} \mathrm{GeV}$ & $m_{\nu_{2,3 R}} \sim 10^{14} \mathrm{GeV}$ & $m_{C} \sim 10^{14} \mathrm{GeV}$ \\
Large-field inflation & $g_{N} \sim f_{22,33}^{\nu} \lesssim 10^{-2}$ & $f_{11}^{\nu} \sim 10^{-5}$ & $\lambda \sim 10^{-8}$ & $\lambda_{10,11,12} \sim 10^{-10}$ \\
& $m_{\Phi} \simeq 2.8 \times 10^{13} \mathrm{GeV}$ & $m_{\nu_{1 R}} \sim 10^{12} \mathrm{GeV}$ & $m_{\nu_{2,3 R}} \lesssim 10^{15} \mathrm{GeV}$ & $m_{C} \lesssim 10^{15} \mathrm{GeV}$ \\
Neutrino and DM masses & $m_{\nu} \sim 0.1 \mathrm{eV}$ & $m_{N} \sim 1 \mathrm{GeV}$ & $m_{H^{\prime}} \sim 1 \mathrm{GeV}$ & $m_{W_{L}^{\prime}} \sim 2.5 \mathrm{TeV}$ \\
\hline \hline
\end{tabular}

The couplings $x=V_{e L}^{\dagger} h^{\nu} V_{\nu R}$ and $y=V_{N L}^{\dagger} h^{\nu} V_{\nu R}$ are generally distinct and complex, and hence sources of $C P$ violation. In addition, we have $x=V^{\prime} y$ and $x^{\dagger} x=y^{\dagger} y$, where $V^{\prime}=V_{e L}^{\dagger} V_{N L}$ plays a role similar to the ordinary lepton and quark mixing matrices.

Notice that the right-handed neutrinos can decay (out of thermal equilibrium) simultaneously into

(a) normal matter: $\nu_{k R} \rightarrow \eta_{2} e_{i}$,

(b) dark matter: $\nu_{k R} \rightarrow \eta_{3} N_{i}$,

which subsequently, due to the $W$-parity conservation, generate two different and unrelated $C P$ asymmetries, $\epsilon_{\mathrm{SM}}^{i}$ and $\epsilon_{\mathrm{DM}}^{i}$, given, respectively, as

$$
\begin{gathered}
\epsilon_{\mathrm{SM}}^{i}=\frac{\Gamma\left(\nu_{k R} \rightarrow \eta_{2} e_{i}\right)-\Gamma\left(\nu_{k R} \rightarrow \bar{\eta}_{2} \bar{e}_{i}\right)}{\Gamma_{\nu_{k R}}}, \\
\epsilon_{\mathrm{DM}}^{i}=\frac{\Gamma\left(\nu_{k R} \rightarrow \eta_{3} N_{i}\right)-\Gamma\left(\nu_{k R} \rightarrow \bar{\eta}_{3} \bar{N}_{i}\right)}{\Gamma_{\nu_{k R}}},
\end{gathered}
$$

via the Feynman diagrams as depicted in Fig. 2, where $\Gamma_{\nu_{k R}}$ is the total width of $\nu_{k R}$, with assuming that $M_{k}<M_{j}$ for all $j \neq k$ (for a fixed $k$, and often chosen $k=1$ as in the previous section). The dark matter production $\left(\eta_{3} N\right)$ is a new observation of this work. Furthermore, it is checked that all other new particles including $W^{\prime \prime}$ negligibly contribute to the $C P$ asymmetries, which contrast with Ref. [28]. We thus obtain

$$
\begin{aligned}
\epsilon_{\mathrm{SM}}^{i} & =\frac{1}{16 \pi\left(x^{\dagger} x\right)_{k k}} \sum_{j \neq k} \Im\left[\left(x^{\dagger} x\right)_{j k} x_{i j}^{*} x_{i k}\right] g\left(\xi_{j k}\right), \\
\epsilon_{\mathrm{DM}}^{i} & =\frac{1}{16 \pi\left(y^{\dagger} y\right)_{k k}} \sum_{j \neq k} \mathfrak{s}\left[\left(y^{\dagger} y\right)_{j k} y_{i j}^{*} y_{i k}\right] g\left(\xi_{j k}\right),
\end{aligned}
$$

where $\xi_{j k}=M_{j}^{2} / M_{k}^{2}$, and

$$
g(\xi)=\sqrt{\xi}\left[\frac{2}{1-\xi}+1-(1+\xi) \ln \frac{1+\xi}{\xi}\right]
$$

We stress that the ordinary leptons $\left(e_{i}\right)$ each carry a distinct flavor number, $L_{i}\left(e_{i}\right)=1$, and the $C P$ asymmetries
$\epsilon_{\mathrm{SM}}^{i}$ are often thought to depend on flavor [63], i.e., each of them creating a separate contribution to the baryon asymmetry, $\eta_{\mathrm{SM}}^{i}$, via the electroweak sphaleron. However, this flavor effect does not hold here for the case of large-field inflation since the largest interaction rate corresponding to the tau flavor is $\Gamma_{\tau} \simeq 5 \times 10^{-3}\left(h^{\tau}\right)^{2} T$, is still slower than the cosmological expansion rate $(H)$ for $T=M_{k} \sim 10^{12} \mathrm{GeV}$, which is just $m_{\nu_{1 R}}$ as listed in Table IV [64]. In this case, the lepton asymmetry has no knowledge of flavor and the net contribution is simply summed as follows

$$
\begin{aligned}
\epsilon_{\mathrm{SM}} & =\sum_{i} \epsilon_{\mathrm{SM}}^{i} \\
& =\frac{1}{16 \pi\left(h^{\nu \dagger} h^{\nu}\right)_{k k}} \sum_{j \neq k} \mathfrak{s}\left[\left(h^{\nu \dagger} h^{\nu}\right)_{j k}^{2}\right] g\left(\xi_{j k}\right),
\end{aligned}
$$

where it is sufficient for us, for simplicity, to take $V_{\nu R}=1$.
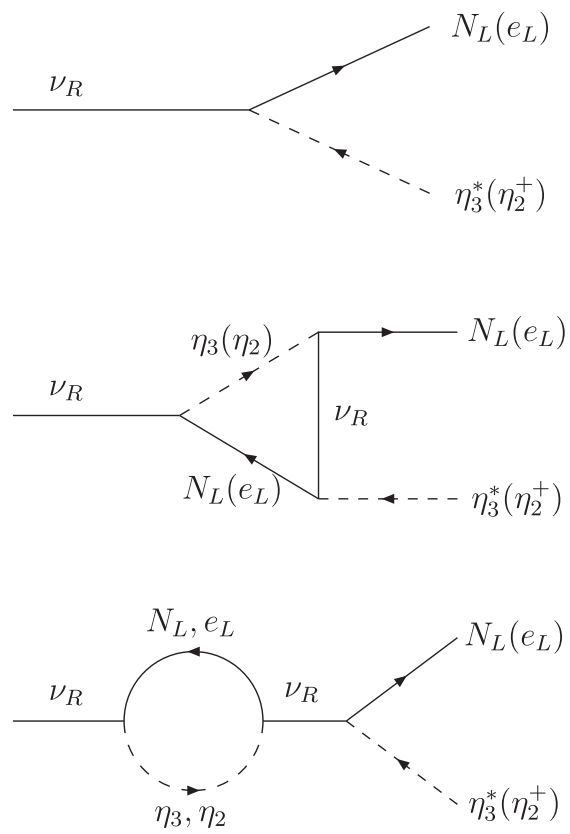

FIG. 2. $C P$-asymmetric decays of $\nu_{R}$ into dark matter $\left(N \eta_{3}\right)$ and normal matter $\left(e \eta_{2}\right)$, respectively, where the Feynman rules and flavor indices can be extracted from Eq. (38). 
However, the wrong particles $N_{i}$ have $h^{N_{i}}$ as Yukawa couplings to the new Higgs $\chi$, and we assume $h^{N_{i}} \gg h^{\tau}$ for some $i$. Thus, the interaction rate of $N_{i}, \Gamma_{N_{i}} \simeq$ $5 \times 10^{-3}\left(h^{N_{i}}\right)^{2} T$, is much faster than the Hubble rate during the time of the $N_{i}$ asymmetry production, $T=M_{k} \sim$ $10^{12} \mathrm{GeV}$. In other words, flavor effects should be taken into account for the $N_{i}$ asymmetric relic. Considering the number of active flavors $n_{f}=2$, i.e. $h^{N_{1,2}} \sim h^{\tau} \ll h^{N_{3}}$, the dark matter asymmetry is given by $\epsilon_{\mathrm{DM}}=\epsilon_{\mathrm{DM}}^{3}+\epsilon_{\mathrm{DM}}^{2^{\prime}}$, where the flavor washout factors approach unit for the large $h^{\nu}$ couplings, and $2^{\prime}$ is some flavor combination of $i=1,2$ orthogonal to 3 . Taking $2^{\prime}=2$, we get, without loss of generality,

$$
\begin{aligned}
\epsilon_{\mathrm{DM}} & =\frac{1}{16 \pi\left(h^{\nu^{\dagger}} h^{\nu}\right)_{k k}} \sum_{i=2,3} \sum_{j \neq k} \sum_{l, l^{\prime}=1,2,3} \Im\left[\left(h^{\nu^{\dagger}} h^{\nu}\right)_{j k} h_{l j}^{\nu *} h_{l^{\prime} k}^{\nu} V_{l^{\prime} i}^{*} V_{l i}\right] g\left(\xi_{j k}\right) \\
& =2 \epsilon_{\mathrm{SM}}+\frac{1}{16 \pi\left(h^{\nu^{\dagger}} h^{\nu}\right)_{k k}} \sum_{i=2,3} \sum_{j \neq k} \sum_{l \neq l^{\prime}} \Im\left[\left(h^{\nu \dagger} h^{\nu}\right)_{j k} h_{l j}^{\nu *} h_{l^{\prime} k}^{\nu} V_{l^{\prime} i}^{*} V_{l i}\right] g\left(\xi_{j k}\right),
\end{aligned}
$$

for fixed $k$ and $V \equiv V_{N L}$. It is clear that the dark matter asymmetry recovers the unflavored standard model values for $l=l^{\prime}$, and the $l \neq l^{\prime}$ terms are also of the same order. We conclude that $\epsilon_{\mathrm{DM}} \sim \epsilon_{\mathrm{SM}}$, i.e., the flavor effect only separates the normal and dark matter asymmetries. Remark: When the flavor effects are neglected, i.e., $h^{N_{i}} \lesssim h^{\tau}$ for all $i$, the dark matter asymmetry is thus summed over several favors, $\epsilon_{\mathrm{DM}}=\sum_{i=1,2,3} \epsilon_{\mathrm{DM}}^{i}=\epsilon_{\mathrm{SM}}$, which is the same as the lepton asymmetry. This case also applies for the $\eta_{3}$ production when it is the lightest $W$ particle.

However, the wrong particles $N_{i}$ and $\eta_{3}$ are singlets under the standard model symmetry. Thus, the $C P$ asymmetries $\epsilon_{\mathrm{DM}}^{i}$ are not affected by the electroweak sphaleron nor do they contribute to the baryon asymmetry, as ensured by $W$-parity conservation. The Boltzmann equations can be split into two, one given by the conventional computation for the lepton asymmetry $\epsilon_{\mathrm{SM}}=\sum_{i=1,2,3} \epsilon_{\mathrm{SM}}^{i}$ responsible for the baryon asymmetry while the other, given as $\epsilon_{\mathrm{DM}}=\sum_{i=2,3} \epsilon_{\mathrm{DM}}^{i}$, is responsible for the dark matter asymmetry $\left(\eta_{\mathrm{DM}}\right)$. (Here one assumes $N_{i}$ to be lighter than $\eta_{3}$, the inverse case is briefly discussed below).

Therefore, the total matter asymmetry of the universe originating from leptogenesis contains several asymmetries as

$$
\eta_{M}=\eta_{B}+\eta_{\mathrm{DM}}
$$

where

$$
\eta_{B}=-\frac{8}{15} \sum \eta_{\mathrm{SM}}
$$

As analyzed, the sphaleron converts only ordinary leptons to ordinary baryons, and this does not work for dark matter, since $L(N)=0$. The latter also holds for either $L(N) \neq 0$ (i.e., $n \neq 0$ ) or $\eta_{3}$ is the lightest $W$ particle, because in this case heavier $W$ particles such as exotic quarks $\left(j_{i}\right)$ or new gauge and scalar fields $\left(W^{\prime \prime}, \chi_{2}, \rho_{3}\right)$ may be createdconverted from the $N$ or $\eta_{3}$ asymmetries via $S U(3)_{L}$ sphaleron processes that preserve $W$ parity. However, they will decay back to the dark matter, since there is no way to keep them stable, in contrast to the case of ordinary baryons. The total contribution of the two decay modes allows us to explain successfully the baryon and dark matter relics through thermal leptogenesis, as shown below.

As explicitly pointed out in the Appendix, the numerical investigation for $\eta_{\mathrm{SM}}$ vs $\eta_{\mathrm{DM}}$ given in Eqs. (44) and (45) for various choices of $V_{l i}$ in the region $5 \times 10^{-11}<\eta_{B}<$ $10^{-10}$ always yields that the asymmetries in the two decay channels end up of the same order, $\eta_{\mathrm{SM}} \sim \eta_{\mathrm{DM}}$. On the other hand, the asymmetries are required to reproduce the observed baryon and dark matter abundance. The ratio of the dark matter and baryon density $\Omega_{\mathrm{DM}} / \Omega_{B}$ is proportional to that of the asymmetries, $\Omega_{\mathrm{DM}} / \Omega_{B}=$ $\eta_{\mathrm{DM}} m_{\mathrm{DM}} /\left(\eta_{B} m_{p}\right)[65,66]$. Since $\eta_{\mathrm{DM}} \sim \eta_{B}$, the dark matter mass is $m_{\mathrm{DM}} \sim m_{p}$ (the proton mass), so as to fit the observed ratio $\Omega_{\mathrm{DM}} \sim 5 \Omega_{B}$.

For the case of small-field inflation, the lepton and dark matter asymmetries can all depend on flavor, because the temperature at asymmetric production $T=M_{k} \sim$ $10^{10} \mathrm{GeV}$ (just $m_{\nu_{1 R}}$ in Table IV) is low. In this case, both $\Gamma_{\tau}$ and $\Gamma_{N_{3}}$ (even $\Gamma_{N_{1,2}}$ if included) are faster than the Hubble rate, i.e., the Yukawa interactions of $\tau$ and $N_{3}$ are in thermal equilibrium. Similarly to the previous case, we obtain the same conclusion $\eta_{\mathrm{SM}} \sim \eta_{\mathrm{DM}}$, i.e., $m_{\mathrm{DM}} \sim m_{p}$, because all the asymmetries are proportional to the unflavored contribution (44).

There may be a case when $\nu_{k R}$ is produced directly from the inflaton decay $\Phi \rightarrow \nu_{k R} \nu_{k R}$, with the matter asymmetries followed by a nonthermal leptogenesis. The total $C P$ asymmetry is simply the sum over all flavor $C P$ asymmetries, $\epsilon_{\mathrm{SM}}=\sum_{i=1,2,3} \epsilon_{\mathrm{SM}}^{i}$ and $\epsilon_{\mathrm{DM}}=\sum_{i=1,2,3} \epsilon_{\mathrm{DM}}^{i}$, which yields $\epsilon_{\mathrm{SM}}=\epsilon_{\mathrm{DM}}$. The lepton and dark matter asymmetries are related to the $C P$ asymmetries by $\eta_{L, D M}=\frac{3}{2} \epsilon_{S M, D M} \times$ $\operatorname{Br}\left(\Phi \rightarrow \nu_{k R} \nu_{k R}\right) \times \frac{T_{R}}{m_{\Phi}}$, respectively, which leads to $\eta_{L}=\eta_{\mathrm{DM}}$. In order to fit $5 \simeq \Omega_{\mathrm{DM}} / \Omega_{B}=m_{\mathrm{DM}} \eta_{\mathrm{DM}} /$ $\left(m_{p} \eta_{B}\right) \sim m_{\mathrm{DM}} / m_{p}$ one finds that the dark matter relic in the nonthermal case is also light, as above. 
Two remarks are in order:

(1) If $N$ is an asymmetric dark matter, its mass is close to $m_{p}$, yet it can avoid restrictions from electroweak precision tests as well as direct searches because, in contrast to Refs. [26,27,30], it is a standard model singlet, and has only interaction with $Z^{\prime}, Z^{\prime \prime}$.

(2) If $\eta_{3}$ is an asymmetric dark matter, we write it in the physical basis as $\eta_{3}=\left(w H^{\prime}-u W_{L}^{\prime}\right) / \sqrt{w^{2}+u^{2}}$, where $W_{L}^{\prime} \equiv G_{X}^{*}$ [27]. The interaction of $\eta_{3}$ with the leptons (37) yields

$$
\begin{aligned}
& \frac{w}{\sqrt{u^{2}+w^{2}}} h_{a b}^{\nu} \bar{N}_{a L} \nu_{b R} H^{\prime} \\
& -\frac{u}{\sqrt{u^{2}+w^{2}}} h_{a b}^{\nu} \bar{N}_{a L} \nu_{b R} W_{L}^{\prime} .
\end{aligned}
$$

Since $u \ll w$, the first term generates a light asymmetric dark matter candidate $H^{\prime}$ which again, thanks to its standard model singlet nature, can avoid all experimental bounds. However, the second is also consistent with $W_{L}^{\prime}$, the Goldstone of $W^{\prime}$, as a dark matter candidate. In this case it implies $\epsilon_{\mathrm{DM}} \sim(u / w)^{2} \epsilon_{\mathrm{SM}}$, leading to $m_{W_{L}^{\prime}} \sim(w / u)^{2} m_{p} \sim$ $2.5 \mathrm{TeV}$, provided that $u \sim 100 \mathrm{GeV}$ and $w \sim 5 \mathrm{TeV}$. This result agrees with Ref. [66].

We conclude that in our scenario the asymmetric dark matter may be a light fermion or scalar state $\left(m_{N, H^{\prime}} \sim \mathrm{GeV}\right)$ transforming as a standard model singlet, or a heavy vector state $\left(m_{W_{L}^{\prime}} \sim 2.5 \mathrm{TeV}\right)$ transforming as a standard model doublet. The dark matter is produced by the standard thermal or nonthermal leptogenesis mechanism. We emphasize that our dark matter phenomenology would then significantly differ from the previous ones discussed in the literature [67-69].

\section{CONCLUSION}

We have pointed out that the seesaw scenario with noncommuting $B-L$ dynamics can successfully address several of the leading cosmological challenges of the standard model. Our proposal provides a common theoretical setup for the generation of neutrino mass, dark matter, inflation, and baryon asymmetry, from first principles. The seesaw mechanism is based on the noncommutative $B-L$ gauge symmetry present in a 3-3-1-1 standard model extension. The latter implies a conserved matter parity that stabilizes dark matter, which is manifestly unified with normal matter. Inflation is driven by the $B-L$ breaking field, with appropriate $\Lambda$ scale and $g_{N}$ coupling. On the other hand, leptogenesis consistently generates not only the present-day baryon asymmetry, but also the dark matter.

The model can harbor three asymmetric dark matter candidates: a scalar $\left(H^{\prime}\right)$ and a fermion $(N)$, both with mass similar to the proton mass, and a vector $\left(W^{\prime}\right)$ with mass at the TeV scale. Although strange $a$ priori, we note that the restrictions coming from electroweak precision studies as well as those arising from collider and direct dark matter searches can be avoided in the former case, while $\mathrm{TeV}$ masses do indeed match the model parameters expected in the second case.

Note also that our scenario may be potentially viable in other gauge groups containing noncommutative $B-L$, such as $S U(3)_{C} \otimes S U(P)_{L} \otimes U(1)_{X} \otimes U(1)_{N}$ for $P \geq 3$, and $S U(3)_{C} \otimes S U(N)_{L} \otimes S U(M)_{R} \otimes U(1)_{X}$ for $(N, M)=$ $(2,3),(3,2),(3,3), \cdots$ Here, the first model class yields a consistent inflation scenario due to the small $g_{N}$, whereas the latter ones provide successful inflation schemes only if the inflaton, which breaks $B-L$ with a large strength, couples nonminimally to gravity. Likewise, the procedure should hold also for $S O(10)$ and trinification models.

\section{ACKNOWLEDGMENTS}

This work is funded by the Vietnam National Foundation for Science and Technology Development (NAFOSTED) under Grant No. 103.01-2016.77. D. C. and F. S. Q. are funded by UFRN and MEC. F. S. Q. also acknowledges financial support from ICTP-SAIFR FAPESP Grant No. 2016/01343-7. J. W. F. V. is funded by Spanish Grants No. FPA2017-85216-P (AEI/FEDER, UE), No. SEV-2014-0398, and No. PROMETEO/2018/165 (Generalitat Valenciana) and the Spanish Red Consolider MultiDark FPA2017-90566-REDC.

\section{APPENDIX: THE $\eta_{\mathrm{DM}} / \boldsymbol{\eta}_{B}$ RATIO}

We first diagonalize the light neutrino mass matrix as obtained from the seesaw mechanism, $V_{\nu}^{T} m_{\nu} V_{\nu}=$ $\operatorname{diag}\left(m_{1}, m_{2}, m_{3}\right)$ [8,9]. The lepton mixing matrix is defined by $V=V_{e L}^{\dagger} V_{\nu}$, which can generally be parametrized by three mixing angles $\left(\theta_{i j}\right)$ and three $C P$-violation phases $(\delta, \sigma, \rho)$,

$$
V=\left(\begin{array}{ccc}
c_{12} c_{13} & s_{12} c_{13} & s_{13} e^{-i \delta} \\
-s_{12} c_{23}-c_{12} s_{23} s_{13} e^{i \delta} & c_{12} c_{23}-s_{12} s_{23} s_{13} e^{i \delta} & s_{23} c_{13} \\
s_{12} s_{23}-c_{12} c_{23} s_{13} e^{i \delta} & -c_{12} s_{23}-s_{12} c_{23} s_{13} e^{i \delta} & c_{23} c_{13}
\end{array}\right) P
$$




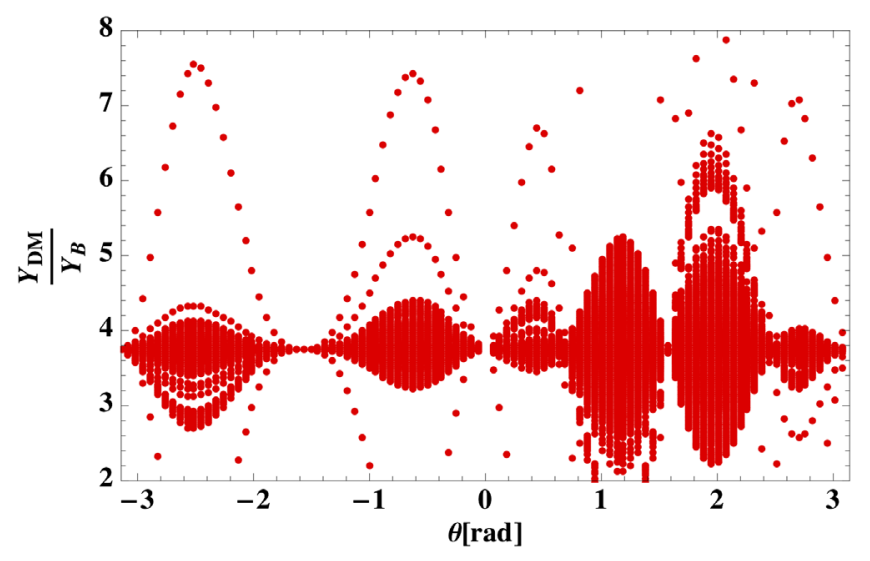

FIG. 3. The ratio $\eta_{\mathrm{DM}} / \eta_{B}$ varies as function of $\theta$ when changing the parameter $\hat{\theta}$.

where $s_{i j}=\sin \theta_{i j}, c_{i j}=\cos \theta_{i j}$, and $P=\operatorname{diag}\left(1, e^{i \sigma / 2}, e^{i \rho / 2}\right)$ follows the PDG description, where the two Majorana phases are placed as a diagonal matrix, while $\delta$ is the Dirac phase. We use the experimental numbers

$$
\begin{gathered}
s_{12}^{2}=0.307 \pm 0.013, \quad s_{23}^{2}=0.417_{-0.028}^{+0.025}, \\
s_{13}^{2}=(2.12 \pm 0.08) \times 10^{-2}, \\
\Delta m_{21}^{2}=m_{2}^{2}-m_{1}^{2}=(7.53 \pm 0.18) \times 10^{-5} \mathrm{eV}^{2},
\end{gathered}
$$

$$
\Delta m_{32}^{2}=m_{3}^{2}-m_{2}^{2}=(2.51 \pm 0.05) \times 10^{-3} \mathrm{eV}^{2}
$$

Without loss of generality, we suppose the ordinary charged leptons to be flavor diagonal, i.e., $V_{e L}=I$, thus $V_{\nu}=V$.

As in the text, we assume $f^{\nu}=\operatorname{diag}\left(f_{11}^{\nu}, f_{22}^{\nu}, f_{33}^{\nu}\right)$, then $M=\operatorname{diag}\left(m_{\nu_{1 R}}, m_{\nu_{2 R}}, m_{\nu_{3 R}}\right) \equiv \operatorname{diag}\left(M_{1}, M_{2}, M_{3}\right)$. Following Ref. [70], the general coupling matrix is given by

$$
\begin{aligned}
h^{\nu}= & \frac{\sqrt{2}}{u} \operatorname{diag}\left(\sqrt{M_{1}}, \sqrt{M_{2}}, \sqrt{M_{3}}\right) R \\
& \times \operatorname{diag}\left(\sqrt{m_{1}}, \sqrt{m_{2}}, \sqrt{m_{3}}\right) V_{\nu}^{\dagger},
\end{aligned}
$$

where $R$ is an orthogonal matrix, parametrized in terms of arbitrary complex angles, $\hat{\theta}_{1}, \hat{\theta}_{2}, \hat{\theta}_{3}$,

$R=\left(\begin{array}{ccc}\hat{c}_{2} \hat{c}_{3} & -\hat{c}_{1} \hat{s}_{3}-\hat{s}_{1} \hat{s}_{2} \hat{c}_{3} & \hat{s}_{1} \hat{s}_{3}-\hat{c}_{1} \hat{s}_{2} \hat{c}_{3} \\ \hat{c}_{2} \hat{s}_{3} & \hat{c}_{1} \hat{c}_{3}-\hat{s}_{1} \hat{s}_{2} \hat{s}_{3} & -\hat{s}_{1} \hat{c}_{3}-\hat{c}_{1} \hat{s}_{2} \hat{s}_{3} \\ \hat{s}_{2} & \hat{s}_{1} \hat{c}_{2} & \hat{c}_{1} \hat{c}_{2}\end{array}\right)$

where $\hat{c}_{i}=\cos \hat{\theta}_{i}$ and $\hat{s}_{i}=\sin \hat{\theta}_{i}$ for $i=1,2,3$.

The mixing matrix of fermion dark matter can be parametrized similarly to the lepton mixing matrix as follows:

$$
V_{N_{R}}=I, \quad V_{N_{L}}=\left(\begin{array}{ccc}
c_{12}^{\prime} c_{13}^{\prime} & s_{12}^{\prime} c_{13}^{\prime} & s_{13}^{\prime} e^{-i \delta^{\prime}} \\
-s_{12}^{\prime} c_{23}^{\prime}-c_{12}^{\prime} s_{23}^{\prime} s_{13}^{\prime} e^{i \delta^{\prime}} & c_{12}^{\prime} c_{23}^{\prime}-s_{12}^{\prime} s_{23}^{\prime} s_{13}^{\prime} e^{i \delta^{\prime}} & s_{23}^{\prime} c_{13}^{\prime} \\
s_{12}^{\prime} s_{23}^{\prime}-c_{12}^{\prime} c_{23}^{\prime} s_{13}^{\prime} e^{i \delta^{\prime}} & -c_{12}^{\prime} s_{23}^{\prime}-s_{12}^{\prime} c_{23}^{\prime} s_{13}^{\prime} e^{i \delta^{\prime}} & c_{23}^{\prime} c_{13}^{\prime}
\end{array}\right),
$$

where $c_{i j}^{\prime}=\cos \theta_{i j}^{\prime}, s_{i j}^{\prime}=\sin \theta_{i j}^{\prime}$.
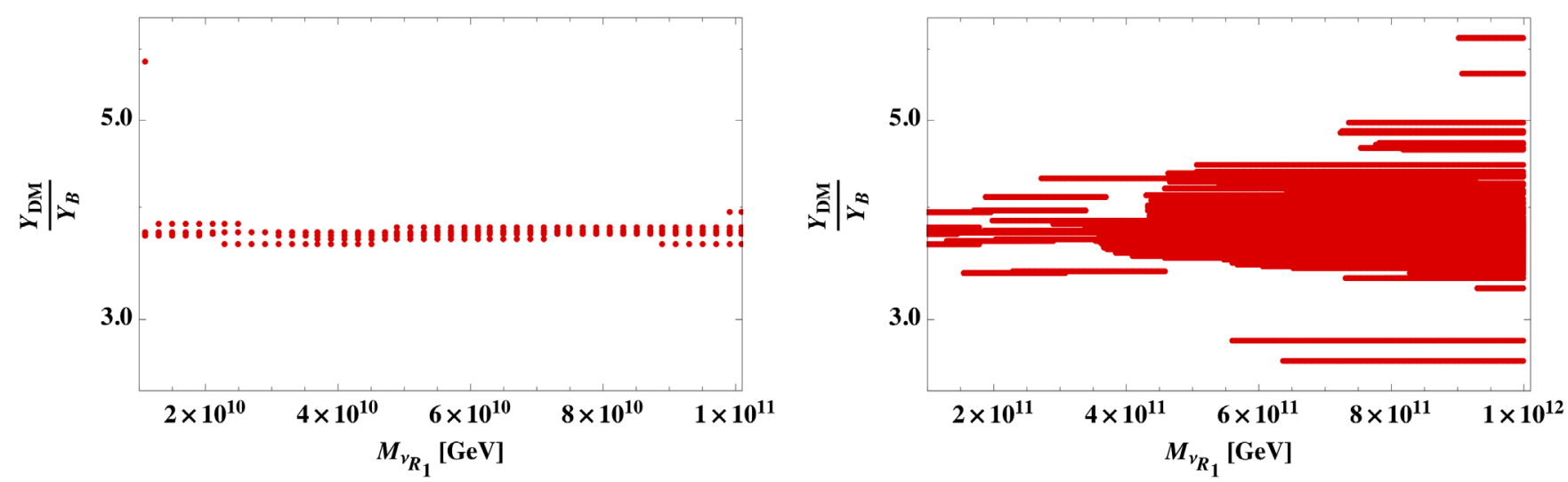

FIG. 4. The ratio $\eta_{\mathrm{DM}} / \eta_{B}$ varies as function of $m_{\nu_{1 R}}$ when changing the remaining parameters. 
When the generation of normal and dark matter is flavor independent, we have $\epsilon_{\mathrm{DM}}=\epsilon_{\mathrm{SM}}$ (thus $\eta_{\mathrm{DM}} \sim \eta_{B}$ ). Hence, the normal and dark matter asymmetries are only separated due to flavor effects, which depend on the $m_{\nu_{1 R}}$ and Yukawa couplings. Without loss of generality, we can study the magnitude of the asymmetry separation for a fixed, e.g., $m_{\nu_{1 R}}=10^{12} \mathrm{GeV}$, where the dependence on the two flavors happens. For this purpose, we plot $\eta_{\mathrm{DM}} / \eta_{B}$ in the measured region of $5 \times 10^{-11}<\eta_{B}<10^{-10}$, provided that $m_{\nu_{2 R}}=m_{\nu_{3 R}}=10^{2} m_{\nu_{1 R}}, \hat{\theta}_{1}=\hat{\theta}_{2}=\hat{\theta}_{3} \equiv \hat{\theta}$ with $-5 \leq$ $\operatorname{Im}[\hat{\theta}] \leq 5,-20 \leq \operatorname{Re}[\hat{\theta}] \leq 20, \quad-\pi \leq \theta_{12}^{\prime}=\theta_{13}^{\prime}=\theta_{23}^{\prime} \equiv$ $\theta \leq \pi, \delta^{\prime}=0$. The ratio $\frac{\eta_{\mathrm{DM}}}{\eta_{\mathrm{SM}}}$ varies as function of $\theta$ for changing the remaining parameter $\hat{\theta}$, as presented in Fig. 3 .

Finally, one can perform a numerical study when changing the lightest right-handed neutrino mass in the range $10^{10} \mathrm{GeV}<m_{\nu_{1 R}}<10^{12} \mathrm{GeV}$, which again implies that the normal and dark matter asymmetries are still viable and satisfy $\eta_{\mathrm{DM}} \sim \eta_{B}$. Indeed, we continue investigating the region $5 \times 10^{-11}<\eta_{B}<10^{-10}$ with assumption $m_{\nu_{2 R}}=$ $m_{\nu_{3 R}}=10^{2} m_{\nu_{1 R}}$, for $10^{10} \mathrm{GeV} \leq m_{\nu_{R_{1}}} \leq 10^{12} \mathrm{GeV}, \hat{\theta}_{1}=$ $\hat{\theta}_{2}=\hat{\theta}_{3} \equiv \hat{\theta}$ with $-\pi \leq \operatorname{Im}[\hat{\theta}] \leq \pi, \operatorname{Re}[\hat{\theta}]=-15, \quad-\pi \leq$ $\theta_{12}^{\prime}=\theta_{13}^{\prime}=\theta_{23}^{\prime} \equiv \theta \leq \pi, \quad \delta^{\prime}=0$. The ratio $\eta_{\mathrm{DM}} / \eta_{B}$ as a function of the lightest right-handed neutrino mass is shown in panels in Fig. 4. It is difficult to generate the dark matter and normal matter asymmetries if $m_{\nu_{1 R}}<10^{10} \mathrm{GeV}$.

Let us remind the reader that $\eta_{B, D M}$ generally depend on the $C P$ phases $\delta, \sigma, \rho, \delta^{\prime}$ and the complex angles $\hat{\theta}_{1,2,3}$. However, these asymmetries are not very sensitive to the $C P$ phases (thus set as 0 ) or pure real $\hat{\theta}_{1,2,3}$ (thus fixed for the second case), while they change significantly as functions of pure imaginary $\hat{\theta}_{1,2,3}$. The thermal leptogenesis requires the reheating temperature to be adequately large $T_{R} \sim m_{\nu_{1 R}}$, but for the nonthermal leptogenesis $T_{R}$ may be much lower than $m_{\nu_{1 R}}$.
[1] P. Minkowski, $\mu \rightarrow e \gamma$ at a rate of one out of $10^{9}$ Muon decays? Phys. Lett. B 67, 421 (1977).

[2] M. Gell-Mann, P. Ramond, and R. Slansky, Complex spinors and unified theories, Conf. Proc. C790927, 315 (1979).

[3] T. Yanagida, Horizontal symmetry and masses of neutrinos, Conf. Proc. C7902131, 95 (1979).

[4] S. L. Glashow, The future of elementary particle physics, NATO Sci. Ser. B 61, 687 (1980).

[5] R. N. Mohapatra and G. Senjanovic, Neutrino Mass and Spontaneous Parity Violation, Phys. Rev. Lett. 44, 912 (1980).

[6] R. N. Mohapatra and G. Senjanovic, Neutrino masses and mixings in gauge models with spontaneous parity violation, Phys. Rev. D 23, 165 (1981).

[7] G. Lazarides, Q. Shafi, and C. Wetterich, Proton lifetime and Fermion masses in an SO(10) model, Nucl. Phys. B181, 287 (1981).

[8] J. Schechter and J. W. F. Valle, Neutrino masses in $\mathrm{SU}(2) \times \mathrm{U}(1)$ theories, Phys. Rev. D 22, 2227 (1980).

[9] J. Schechter and J. W. F. Valle, Neutrino decay and spontaneous violation of lepton number, Phys. Rev. D 25, 774 (1982).

[10] M. Fukugita and T. Yanagida, Baryogenesis without grand unification, Phys. Lett. B 174, 45 (1986).

[11] W. Buchmuller, R. D. Peccei, and T. Yanagida, Leptogenesis as the origin of matter, Annu. Rev. Nucl. Part. Sci. 55, 311 (2005).

[12] S. Davidson, E. Nardi, and Y. Nir, Leptogenesis, Phys. Rep. 466, 105 (2008).

[13] A. A. Starobinsky, A new type of isotropic cosmological models without singularity, Phys. Lett. B 91, 99 (1980).
[14] A. H. Guth, The inflationary Universe: A possible solution to the horizon and flatness problems, Phys. Rev. D 23, 347 (1981).

[15] C. Patrignani et al. (Particle Data Group), Review of particle physics, Chin. Phys. C 40, 100001 (2016).

[16] G. Jungman, M. Kamionkowski, and K. Griest, Supersymmetric dark matter, Phys. Rep. 267, 195 (1996).

[17] G. Bertone, D. Hooper, and J. Silk, Particle dark matter: Evidence, candidates and constraints, Phys. Rep. 405, 279 (2005).

[18] F. Iocco, M. Pato, and G. Bertone, Evidence for dark matter in the inner Milky Way, Nat. Phys. 11, 245 (2015).

[19] B. S. Acharya et al. (Cherenkov Telescope Array Consortium Collaboration), Science with the Cherenkov telescope array, arXiv:1709.07997.

[20] M. Tavani et al. (e-ASTROGAM Collaboration), Science with e-ASTROGAM (A space mission for $\mathrm{MeV}-\mathrm{GeV}$ gamma-ray astrophysics), J. High Energy Astrophys. 19, 1 (2018).

[21] G. Arcadi, M. Dutra, P. Ghosh, M. Lindner, Y. Mambrini, M. Pierre, S. Profumo, and F. S. Queiroz, The waning of the WIMP? A review of models, searches, and constraints, Eur. Phys. J. C 78, 203 (2018).

[22] J. C. Montero and B. L. Sanchez-Vega, Neutrino masses and the scalar sector of a B-L extension of the standard model, Phys. Rev. D 84, 053006 (2011).

[23] K. Schmitz, The B-L phase transition: Implications for cosmology and neutrinos, Ph.D. thesis, Hamburg University, 2012.

[24] B. L. Snchez-Vega, J. C. Montero, and E. R. Schmitz, Complex scalar DM in a B-L model, Phys. Rev. D 90, 055022 (2014).

[25] W. Rodejohann and C. E. Yaguna, Scalar dark matter in the BL model, J. Cosmol. Astropart. Phys. 12 (2015) 032. 
[26] P. V. Dong, H. T. Hung, and T. D. Tham, 3-3-1-1 model for dark matter, Phys. Rev. D 87, 115003 (2013).

[27] P. V. Dong, D. T. Huong, F. S. Queiroz, and N. T. Thuy, Phenomenology of the 3-3-1-1 model, Phys. Rev. D 90, 075021 (2014).

[28] D. T. Huong, P. V. Dong, C. S. Kim, and N. T. Thuy, Inflation and leptogenesis in the 3-3-1-1 model, Phys. Rev. D 91, 055023 (2015).

[29] D. T. Huong and P. V. Dong, Neutrino masses and superheavy dark matter in the 3-3-1-1 model, Eur. Phys. J. C 77, 204 (2017).

[30] A. Alves, G. Arcadi, P. V. Dong, L. Duarte, F. S. Queiroz, and J. W. F. Valle, Matter-parity as a residual gauge symmetry: Probing a theory of cosmological dark matter, Phys. Lett. B 772, 825 (2017).

[31] P. V. Dong, Unifying the electroweak and B-L interactions, Phys. Rev. D 92, 055026 (2015).

[32] P. V. Dong and D. T. Si, Kinetic mixing effect in the 3-3-1-1 model, Phys. Rev. D 93, 115003 (2016).

[33] M. Singer, J. W. F. Valle, and J. Schechter, Canonical neutral current predictions from the weak electromagnetic gauge group SU(3) X $u$ (1), Phys. Rev. D 22, 738 (1980).

[34] J. W. F. Valle and C. A. Vaquera-Araujo, Dynamical seesaw mechanism for Dirac neutrinos, Phys. Lett. B 755, 363 (2016).

[35] S. Nussinov, Technocosmology: Could a technibaryon excess provide a 'natural' missing mass candidate?, Phys. Lett. 165B, 55 (1985).

[36] K. M. Zurek, Asymmetric dark matter: Theories, signatures, and constraints, Phys. Rep. 537, 91 (2014).

[37] K. Petraki and R. R. Volkas, Review of asymmetric dark matter, Int. J. Mod. Phys. A 28, 1330028 (2013).

[38] S. M. Boucenna, S. Morisi, Q. Shafi, and J. W. F. Valle, Inflation and majoron dark matter in the seesaw mechanism, Phys. Rev. D 90, 055023 (2014).

[39] S. R. Coleman and E. J. Weinberg, Radiative corrections as the origin of spontaneous symmetry breaking, Phys. Rev. D 7, 1888 (1973).

[40] G. Barenboim, E. J. Chun, and H. M. Lee, ColemanWeinberg inflation in light of Planck, Phys. Lett. 730B, 81 (2014).

[41] M. U. Rehman and Q. Shafi, Higgs inflation, quantum smearing and the tensor to scalar ratio, Phys. Rev. D 81, 123525 (2010).

[42] M. U. Rehman, Q. Shafi, and J. R. Wickman, GUT inflation and proton decay after WMAP5, Phys. Rev. D 78, 123516 (2008).

[43] A. D. Linde, Particle physics and inflationary cosmology, Contemp. Concepts Phys. 5, 1 (1990).

[44] G. Hinshaw et al. (WMAP Collaboration), Nine-Year Wilkinson microwave anisotropy probe (WMAP) observations: Cosmological parameter results, Astrophys. J. Suppl. Ser. 208, 19 (2013).

[45] P. A. R. Ade et al. (Planck Collaboration), Planck 2013 results. XVI. Cosmological parameters, Astron. Astrophys. 571, A16 (2014).

[46] P. A. R. Ade et al. (Planck Collaboration), Planck 2015 results. XX. Constraints on inflation, Astron. Astrophys. 594, A20 (2016).
[47] A. R. Liddle and S. M. Leach, How long before the end of inflation were observable perturbations produced?, Phys. Rev. D 68, 103503 (2003).

[48] P. A. R. Ade et al. (BICEP2 and Planck Collaboration), Joint Analysis of BICEP2/Keck?Array and Planck Data, Phys. Rev. Lett. 114, 101301 (2015).

[49] P. A. R. Ade et al. (BICEP2 and Keck Array Collaboration), Improved Constraints on Cosmology and Foregrounds from BICEP2 and Keck Array Cosmic Microwave Background Data with Inclusion of $95 \mathrm{GHz}$ Band, Phys. Rev. Lett. 116, 031302 (2016).

[50] Y. Akrami et al. (Planck Collaboration), Planck 2018 results. X. Constraints on inflation, arXiv:1807.06211.

[51] S. Iso, K. Kohri, and K. Shimada, Small field ColemanWeinberg inflation driven by a fermion condensate, Phys. Rev. D 91, 044006 (2015).

[52] K. Kaneta, O. Seto, and R. Takahashi, Very low scale Coleman-Weinberg inflation with nonminimal coupling, Phys. Rev. D 97, 063004 (2018).

[53] L. Knox and Y.-S. Song, A Limit on the Detectability of the Energy Scale of Inflation, Phys. Rev. Lett. 89, 011303 (2002).

[54] J. R. Ellis, J. S. Hagelin, D. V. Nanopoulos, K. A. Olive, and M. Srednicki, Supersymmetric relics from the big bang, Nucl. Phys. B238, 453 (1984).

[55] V. Berezinsky, M. Kachelriess, and A. Vilenkin, UltrahighEnergy Cosmic Rays without GZK Cutoff, Phys. Rev. Lett. 79, 4302 (1997).

[56] D. J. H. Chung, E. W. Kolb, and A. Riotto, Production of massive particles during reheating, Phys. Rev. D 60, 063504 (1999).

[57] P. Brax, J.-F. Dufaux, and S. Mariadassou, Preheating after small-field inflation, Phys. Rev. D 83, 103510 (2011).

[58] F. L. Bezrukov and M. Shaposhnikov, The Standard Model Higgs boson as the inflaton, Phys. Lett. B 659, 703 (2008).

[59] J. Garcia-Bellido, D. G. Figueroa, and J. Rubio, Preheating in the Standard Model with the Higgs-Inflaton coupled to gravity, Phys. Rev. D 79, 063531 (2009).

[60] L. Kofman, A. D. Linde, and A. A. Starobinsky, Reheating after Inflation, Phys. Rev. Lett. 73, 3195 (1994).

[61] G. N. Felder, L. Kofman, and A. D. Linde, Instant preheating, Phys. Rev. D 59, 123523 (1999).

[62] D. J. H. Chung, E. W. Kolb, and A. Riotto, Nonthermal Supermassive Dark Matter, Phys. Rev. Lett. 81, 4048 (1998).

[63] E. Nardi, Y. Nir, E. Roulet, and J. Racker, The importance of flavor in leptogenesis, J. High Energy Phys. 01 (2006) 164.

[64] J. M. Cline, K. Kainulainen, and K. A. Olive, Protecting the primordial baryon asymmetry from erasure by sphalerons, Phys. Rev. D 49, 6394 (1994).

[65] M. L. Graesser, I. M. Shoemaker, and L. Vecchi, Asymmetric WIMP dark matter, J. High Energy Phys. 10 (2011) 110.

[66] A. Falkowski, J. T. Ruderman, and T. Volansky, Asymmetric dark matter from Leptogenesis, J. High Energy Phys. 05 (2011) 106.

[67] D. Cogollo, A. X. Gonzalez-Morales, F. S. Queiroz, and P.R. Teles, Excluding the light dark matter window of a 331 model using LHC and direct dark 
matter detection data, J. Cosmol. Astropart. Phys. 11 (2014) 002.

[68] C. Kelso, H. N. Long, R. Martinez, and F. S. Queiroz, Connection of $g-2$, , electroweak, dark matter, and collider constraints on 331 models, Phys. Rev. D 90, 113011 (2014).
[69] A. Alves, A. Berlin, S. Profumo, and F. S. Queiroz, Diracfermionic dark matter in $\mathrm{U}(1)_{X}$ models, J. High Energy Phys. 10 (2015) 076.

[70] J. A. Casas and A. Ibarra, Oscillating neutrinos and $\mu \rightarrow e, \gamma$, Nucl. Phys. B618, 171 (2001). 